\title{
Constructing Tight Gabor Frames Using CAZAC Sequences
}

\author{
Mark Magsino \\ Department of Mathematics, University of Maryland \\ 4176 Campus Drive - William E. Kirwin Hall \\ College Park, MD 20742, USA \\ mmagsino@math.umd.edu
}

\begin{abstract}
The construction of finite tight Gabor frames plays an important role in many applications. These applications include significant ones in signal and image processing. We explore when constant amplitude zero autocorrelation (CAZAC) sequences can be used to generate tight Gabor frames. The main theorem uses Janssen's representation and the zeros of the discrete periodic ambiguity function to give necessary and sufficient conditions for determining whether any Gabor frame is tight. The relevance of the theorem depends significantly on the construction of examples. These examples are necessarily intricate, and to a large extent, depend on CAZAC sequences. Finally, we present an alternative method for determining whether a Gabor system yields a tight frame. This alternative method does not prove tightness using the main theorem, but instead uses the Gram matrix of the Gabor system.
\end{abstract}

Key words and phrases: Frames, Gabor Frames, Constant Amplitude Zero Autocorrelation, Time-Frequency Analysis

2010 AMS Mathematics Subject Classification - 42C15, 42C20

\section{Introduction}

1.1. Background. Frames were introduced in 1952 by Duffin and Schaeffer [19] for their work on nonharmonic Fourier series. They used frames to compute the coefficients of a linear combination of vectors which spanned its Hilbert space, but were linearly dependent. Since then, frames have been used in applications such as the analysis of wavelets, and in signal and image processing [17][32][34][38][39]. Frames can be viewed as a generalization of orthonormal bases for Hilbert spaces. In the context of signal processing, the primary advantage of frames is that they provide stable representations of signals which are robust in the presense of erasures and noise [26][27]. Frames for finite vector spaces, i.e., finite frames, are of particular interest for engineering or computational applications, and there has been significant research conducted on finite frames $[1][2][14][15]$. 
Let $\mathcal{H}$ be a separable Hilbert space over the complex field $\mathbb{C}$. A sequence $\mathcal{F}=\left\{v_{i}\right\} \subseteq \mathcal{H}$ is a frame for $\mathcal{H}$ if there exist $A, B>0$ such that

$$
\forall x \in \mathcal{H}, A\|x\|_{2}^{2} \leq \sum_{v_{i} \in \mathcal{F}}\left|\left\langle x, v_{i}\right\rangle\right|^{2} \leq B\|x\|_{2}^{2} .
$$

Since we want to view frames as a generalization of orthonormal bases, we want to be able to write any vector $x \in \mathcal{H}$ in terms of $v_{i} \in \mathcal{F}$. If $\mathcal{F}$ is a frame, then we can write any $x \in \mathcal{H}$ as the linear combination,

$$
x=\sum_{v_{i} \in \mathcal{F}}\left\langle x, v_{i}\right\rangle S^{-1} v_{i}
$$

where $S$ is a well-defined linear operator associated with $\mathcal{F}$ known as the frame operator of $\mathcal{F}$. In general, it is non-trivial to compute the inverse of the frame operator. However, if is possible to have $A=B$, then we have the special case of a tight frame. In this case, $S=A I d$, and (1.2) can be re-written as

$$
x=\frac{1}{A} \sum_{v_{i} \in \mathcal{F}}\left\langle x, v_{i}\right\rangle v_{i} .
$$

This makes tight frames particularly desirable since (1.3) is computationally easier than (1.2). This has motivated research into the discovery and construction of tight frames [7][13][18][40][41], as well as the transformation of non-tight frames into tight frames [28].

Finite frames are sometimes studied in the context of time-frequency analysis. The beginnings of time-frequency analysis can be traced back to Gabor's paper on communication theory [21], where he used time-frequency rectangles to simultaneously analyze the time and frequency content of Gaussian functions. Such methods are restricted by the Heisenberg uncertainty principle, which essentially states that no function can be simultaneously well concentrated in both time and frequency [3][20]. It is this limitation that makes the theory of timefrequency analysis both difficult and mathematically interesting, and there is significant research in the area of time-frequency analysis [8][16][29][36][37].

In particular, systems which consist of translations and modulations of a generating function are called Gabor systems. To ensure any signal (function) can be constructed from a Gabor system, one would like to show that the system forms an orthonormal basis, or more generally, a frame. Gabor's original suggestion was to use Gaussian functions as the generator. His suggestion did not generate a Riesz basis for $L^{2}(\mathbb{R})$ [24], the space of square integrable functions, but a minor alteration to his suggestion does make the system into a frame for $L^{2}(\mathbb{R})$ [31]. This example motivates studying if Gabor systems generated by other functions are tight frames. An excellent reference and exposition on time-frequency analysis is [22].

On the other hand, radar and communication theory often employ special sequences, $\varphi \in \mathbb{C}^{N}$, with the following two properties:

$$
|\varphi[k]|=1, \quad \forall k \in(\mathbb{Z} / N \mathbb{Z})
$$


and

$$
\sum_{k=0}^{N-1} \varphi[k+m] \overline{\varphi[k]}=0, \quad \forall m \in(\mathbb{Z} / N \mathbb{Z}) \backslash\{0\}
$$

The CA property is short for "constant amplitude" and the ZAC property is short for "zero autocorrelation". Sequences which satisfy these properties are known as constant amplitude zero autocorrelation seqeunces, or CAZAC sequences for short.

The central idea is to use CAZAC sequences to generate tight Gabor frames. Radar and communcation theory is closely tied to time-frequency analysis, so it is natural to consider whether sequences they employ could be used to generate tight Gabor frames. In particular, the ZAC property says that time shifts of CAZAC sequences are orthogonal to the original CAZAC sequence. One would hope this property remains when you add frequency shifts to the time shifts, which would make them suitable candidates for tight Gabor frames.

1.2. Outline. In Section 2 we give a necessary and sufficient condition for proving a Gabor system in $\mathbb{C}^{N}$ is a tight frame. This is accomplished through Janssen's representation and by showing sufficient sparsity in the discrete periodic ambiguity function, a tool employed in radar theory [30]. In Section 3, we give a brief overview on constant amplitude zero autocorrelation (CAZAC) sequences and define the CAZAC sequences used in the examples: $\mathrm{Chu}, \mathrm{P} 4$, Wiener, square length Björck-Saffari, and Milewski sequences. Some details on the Chu, P4, and Wiener sequences can be found in [9]. Details on the square length Björck-Saffari sequence, as well as some generalizations of the sequence, can be found in [11]. Details on the Milewski sequence and a detailed computation of its discrete periodic ambiguity function can be found in [6] or [33]. We also provide two alternative formulations of the problem of constructing new CAZAC sequences. In Section 4, we compute the discrete periodic ambiguity functions for the CAZAC sequences described in Section 3. Section 5 constructs several examples which utilize the sequences and computations from Section 4, and Theorem 2.2.

The last two sections are devoted to an alternate approach by Gram matrices. We begin Section 6 by computing the Gram matrix of a Gabor system in terms of the discrete periodic ambiguity function of the generating sequence. We then use the results of Section 4 in order to write the Gram matrices of Gabor systems generated by the $\mathrm{Chu}, \mathrm{P} 4$, and Wiener sequences. Section 7 is presents the alternate method for proving Gabor systems are tight frames. This method proves tightness by showing the Gram matrix has sufficient rank and has only one nonzero eigenvalue. Section 7 primarily focuses on the P4 and Chu sequence cases, but we mention how to make the necessary adjustments for the Wiener sequence case. 
1.3. Notation. Let $\varphi \in \mathbb{C}^{N}$. We denote translation by $k \in(\mathbb{Z} / N \mathbb{Z})$ as $\tau_{k}$ and modulation by $\ell \in(\mathbb{Z} / N \mathbb{Z})$ as $e_{\ell}$, and define them by

$$
\tau_{k}(\varphi)[j]=\varphi[j-k] \text { and } e_{\ell}(\varphi)[j]=e^{2 \pi i j \ell / N} \varphi[j] .
$$

The discrete Fourier transform (DFT), $\widehat{\varphi} \in \mathbb{C}^{N}$, of $\varphi \in \mathbb{C}^{N}$ is defined by

$$
\forall \ell \in(\mathbb{Z} / N \mathbb{Z}), \hat{\varphi}[\ell]=\sum_{k=0}^{N-1} \varphi[k] e^{-2 \pi i k \ell / N} .
$$

In particular, (1.5) is the non-normalized version of the DFT, and so the inverse is given by

$$
\varphi[k]=\frac{1}{N} \sum_{\ell=0}^{N-1} \widehat{\varphi}[\ell] e^{2 \pi i k \ell / N} .
$$

The product group $(\mathbb{Z} / N \mathbb{Z}) \times(\mathbb{Z} / N \mathbb{Z})^{-}$will denote the time-frequency lattice, where $(\mathbb{Z} / N \mathbb{Z})^{-}=\left\{e^{2 \pi i \ell(\cdot) / N}: \ell \in(\mathbb{Z} / N \mathbb{Z})\right\}$. The elements of $(\mathbb{Z} / N \mathbb{Z}) \times(\mathbb{Z} / N \mathbb{Z})^{-}$ are the time-frequency operators $e_{\ell} \tau_{k}$. Since we are working with the product group, the group operation, $*$, will be $e_{\ell} \tau_{k} * e_{n} \tau_{m}=e_{\ell+n} \tau_{k+m}$.

For convenience, we shall write elements of $(\mathbb{Z} / N \mathbb{Z}) \times(\mathbb{Z} / N \mathbb{Z})$ as simply $(k, \ell)$. In particular, here we are using the identification of $((\mathbb{Z} / N \mathbb{Z}) \cdot \cdot)$ with $((\mathbb{Z} / N \mathbb{Z}),+)$, but choose this notation to emphasize that $(\mathbb{Z} / N \mathbb{Z})^{-}$is technically the character group.

In particular, this convention allows us to re-write the product group operation as simply $(k, \ell)+(m, n)=(k+m, \ell+n)$. Throughout we will write that $\Lambda \subseteq(\mathbb{Z} / N \mathbb{Z}) \times(\mathbb{Z} / N \mathbb{Z})^{2}$ is a subgroup in the sense that it will be a subgroup of the product group $(\mathbb{Z} / N \mathbb{Z}) \times(\mathbb{Z} / N \mathbb{Z})$.

\section{Tight frames from sparse discrete periodic ambiguity functions}

The main result of this section, Theorem 2.2, can be summarized as: Gabor systems are tight frames if the discrete periodic ambiguity functions of their generating functions are sufficently sparse. To prove this we use Janssen's representation. Janssen's representation allows us to write the frame operator as a linear combination of time-frequency operators whose coefficients can be computed without knowing the input of the frame operator, cf. Walnut's representation [35][42]. We also draw connections between the discrete periodic ambiguity function and short-time Fourier transform, as well as Theorem 2.2 and the Wexler-Raz theorem. For more details on frame theroy, [15] is a valuable resource.

We begin by defining the ambiguity function, providing associated definitions, and present the main result, Theorem 2.2.

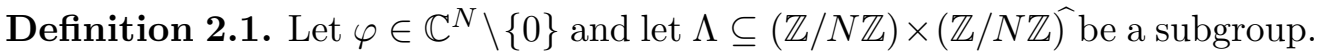


(i) The discrete periodic ambiguity function (DPAF) of $\varphi$ is the function $A_{p}(\varphi):(\mathbb{Z} / N \mathbb{Z}) \times(\mathbb{Z} / N \mathbb{Z})^{\uparrow} \rightarrow \mathbb{C}$ defined by

$$
A_{p}(\varphi)[m, n]=\frac{1}{N} \sum_{k=0}^{N-1} \varphi[k+m] \overline{\varphi[k]} e^{-2 \pi i n k / N}=\frac{1}{N}\left\langle\tau_{-m} \varphi, e_{n} \varphi\right\rangle .
$$

(ii) The adjoint subgroup, $\Lambda^{\circ}$, is the group given by $\Lambda^{\circ}=\{(m, n) \in(\mathbb{Z} / N \mathbb{Z}) \times$ $\left.(\mathbb{Z} / N \mathbb{Z})^{\sim}: e_{n} \tau_{m} e_{\ell} \tau_{k}=e_{\ell} \tau_{k} e_{n} \tau_{m}, \forall(k, \ell) \in \Lambda\right\}$. That is, $\Lambda^{\circ}$ generates all time-frequency operators which commute with all time-frequency operators generated by $\Lambda$.

(iii) $A_{p}(\varphi)$ is $\Lambda^{\circ}$-sparse if for every $(m, n) \in \Lambda^{\circ} \backslash\{(0,0)\}$ we have that $A_{p}(\varphi)[m, n]=0$.

Theorem 2.2. Let $\varphi \in \mathbb{C}^{N} \backslash\{0\}$ and let $\Lambda \subseteq \mathbb{Z} / N \mathbb{Z} \times(\mathbb{Z} / N \mathbb{Z})^{\uparrow}$ be a subgroup. $(\varphi, \Lambda)$ is a tight frame if and only if $A_{p}(\varphi)$ is $\Lambda^{\circ}$-sparse. Moreover, the frame bound is given by $|\Lambda| A_{p}(\varphi)[0,0]$.

Before developing the tools necessary to prove Theorem 2.2, we first discuss the connection of the discrete periodic ambiguity function and the short-time Fourier transform. We also show that full Gabor systems, i.e. ones generated by $\Lambda=(\mathbb{Z} / N \mathbb{Z}) \times(\mathbb{Z} / N \mathbb{Z})$, always give rise to tight frames, independent of the choice of $\varphi$.

Definition 2.3. Let $\varphi \in \mathbb{C}^{N}$. The discrete short-time Fourier transform (STFT) of $\varphi$ with respect to $\psi \in \mathbb{C}^{N}$ is defined by

$$
V_{\psi}(\varphi)[m, n]=\left\langle\varphi, e_{n} \tau_{m} \psi\right\rangle=\sum_{k=0}^{N-1} \varphi[k] \overline{\psi[k-m]} e^{-2 \pi i n k / N}=\left(\varphi \tau_{m}(\bar{\psi}) \hat{)}[n] .\right.
$$

The inversion formula is given by

$$
\varphi[k]=\frac{1}{N\|\psi\|_{2}^{2}} \sum_{n=0}^{N-1} \sum_{m=0}^{N-1} V_{\psi}(\varphi)[m, n] e_{n} \tau_{m} \psi
$$

To connect this to the DPAF, we use the STFT definition to compute,

$$
A_{p}(\varphi)[m, n]=\frac{1}{N}\left\langle\tau_{-m} \varphi, e_{n} \varphi\right\rangle=\frac{e^{2 \pi i m n / N}}{N}\left\langle\varphi, e_{n} \tau_{m} \varphi\right\rangle=\frac{e^{2 \pi i m n / N}}{N} V_{\phi}(\varphi)[m, n] .
$$

Theorem 2.4. Let $\varphi \in \mathbb{C}^{N} \backslash\{0\}$ and let $\Lambda=(\mathbb{Z} / N \mathbb{Z}) \times(\mathbb{Z} / N \mathbb{Z})$. Then, the Gabor system $(\varphi, \Lambda)$ is a tight frame with frame bound $N\|\varphi\|_{2}^{2}$.

Proof. For every $x \in \mathbb{C}^{N}$ we compute $S(x)$,

$$
S(x)=\sum_{n=0}^{N-1} \sum_{m=0}^{N-1}\left\langle x, e_{n} \tau_{m} \varphi\right\rangle e_{n} \tau_{m} \varphi=\sum_{n=0}^{N-1} \sum_{m=0}^{N-1} V_{\varphi}(x)[m, n] e_{n} \tau_{m} \varphi=N\|\varphi\|_{2}^{2} x .
$$

Although Theorem 2.4 does give tight frames, smaller tight Gabor frames would require less coefficients when analyzing signals, and smaller representations of those signals would be possible. Thus, we analyze Gabor systems where $\Lambda$ is a proper subgroup of $(\mathbb{Z} / N \mathbb{Z}) \times(\mathbb{Z} / N \mathbb{Z})$. 
The primary tool in our analysis will be Janssen's representation. Janssen's represenation is a way to express the frame operator entirely in terms of a linear combination of operators that do not require an input function to compute. To make this idea clear, we define the frame operator before presenting Janssen's Theorem. More details on finite frames and the frame operator can be found in the first chapter of [15].

Definition 2.5. Let $\mathcal{H}$ be an $N$-dimensional Hilbert space and let $\mathcal{F}=\left\{v_{i}\right\}_{i=1}^{M} \subseteq$ $\mathcal{H}$ be a frame for $\mathcal{H}$, where $M \geq N$.

(i) The analysis operator $F: \mathcal{H} \rightarrow \mathbb{C}^{M}$ is defined by

$$
\forall x \in \mathcal{H}, F(x)=\left\{\left\langle x, v_{i}\right\rangle\right\}_{i=1}^{M} .
$$

(ii) The adjoint of the analysis operator, $F^{*}: \mathbb{C}^{M} \rightarrow \mathcal{H}$, is called the synthesis operator and is defined by

$$
\forall\left\{c_{i}\right\}_{i=1}^{M} \in \mathbb{C}^{M}, F^{*}\left(\left\{c_{i}\right\}_{i=1}^{M}\right)=\sum_{i=1}^{M} c_{i} v_{i} .
$$

(iii) The frame operator, $S: \mathcal{H} \rightarrow \mathcal{H}$, is defined by $S=F^{*} F$. We can write this explicitly as

$$
\forall x \in \mathcal{H}, S(x)=\sum_{i=1}^{M}\left\langle x, v_{i}\right\rangle v_{i} .
$$

The following form of Janssen's representation is less general than what is usually known as Janssen's representation, but we choose to use this form because it is adjusted for use in Theorem 2.2. A more general version is proved in [35].

Theorem 2.6 (Janssen). Let $\Lambda$ be a subgroup of $(\mathbb{Z} / N \mathbb{Z}) \times(\mathbb{Z} / N \mathbb{Z})^{-}$and $\Lambda^{\circ}$ be the adjoint subgroup of $\Lambda$. Let $\varphi \in \mathbb{C}^{N} \backslash\{0\}$. Then, the Gabor frame operator of the Gabor system $(\varphi, \Lambda)$ can be written as

$$
S=\frac{|\Lambda|}{N} \sum_{(m, n) \in \Lambda^{\circ}}\left\langle e_{n} \tau_{m} \varphi, \varphi\right\rangle e_{n} \tau_{m} .
$$

To prove Theorem 2.2 we require one more theorem. The space of linear operators on $\mathbb{C}^{N}$ forms an $N^{2}$-dimensional space. Moreover, given any orthonormal basis $\left\{e_{i}\right\}_{i=1}^{N}$, we can define the Hilbert-Schmidt inner product of two linear operators $A, B$ by

$$
\langle A, B\rangle_{H S}=\sum_{i=1}^{N} \sum_{j=1}^{N}\left\langle A e_{i}, e_{j}\right\rangle\left\langle B e_{i}, e_{j}\right\rangle .
$$

The Hilbert-Schimdt inner product is independent of choice of orthonormal basis. We call the space of linear operators on $\mathbb{C}^{N}$ equipped with the HilbertSchmidt inner product the Hilbert-Schmidt space. With this in mind, Theorem 2.7 is given without proof, but a proof can be found in [35]. 
Theorem 2.7. The set of normalized time frequency translates $\left\{\frac{1}{\sqrt{N}} e_{\ell} \tau_{k}:(k, \ell) \in\right.$ $\left.(\mathbb{Z} / N \mathbb{Z}) \times(\mathbb{Z} / N \mathbb{Z})^{-}\right\}$forms an orthonormal basis for the $N^{2}$-dimensional HilbertSchmidt space of linear operators on $\mathbb{C}^{N}$.

Equipped with Theorem 2.6 and Theorem 2.7 we now prove Theorem 2.2.

Proof (Theorem 2.2). By Janssen's representation and using the definition of $A_{p}(\varphi)$ we have

$$
\begin{aligned}
S & =\frac{|\Lambda|}{N} \sum_{(k, \ell) \in \Lambda^{\circ}}\left\langle e_{\ell} \tau_{k} \varphi, \varphi\right\rangle e_{\ell} \tau_{k}=\frac{|\Lambda|}{N} \sum_{(k, \ell) \in \Lambda^{\circ}}\left\langle\tau_{k} \varphi, e_{-\ell} \varphi\right\rangle e_{\ell} \tau_{k} \\
& =|\Lambda| \sum_{(k, \ell) \in \Lambda^{\circ}} A_{p}(\varphi)[-k,-\ell] e_{\ell} \tau_{k}=|\Lambda| \sum_{(k, \ell) \in \Lambda^{\circ}} A_{p}(\varphi)[k, \ell] e_{-\ell} \tau_{-k}
\end{aligned}
$$

The last equality comes from re-indexing by replacing $(k, \ell)$ with $(-k,-\ell)$ and using the fact that $\Lambda^{\circ}$ is a subgroup of $(\mathbb{Z} / N \mathbb{Z}) \times(\mathbb{Z} / N \mathbb{Z})^{-}$. Clearly, if $A_{p}(\varphi)$ is $\Lambda^{\circ}$-sparse, then by $(2.6)$ the frame operator will be $|\Lambda| A_{p}(\varphi)[0,0]$ times the identity. It remains to show that $A_{p}(\varphi)$ is a necessary condition. For $S$ to be tight we need

$$
S=|\Lambda| \sum_{(k, \ell) \in \Lambda^{\circ}} A_{p}(\varphi)[k, \ell] e_{\ell} \tau_{k}=A I d .
$$

In particular, we can rewrite (2.7) to

$$
\sum_{(k, \ell) \in \Lambda^{\circ} \backslash\{(0,0)\}}|\Lambda| A_{p}(\varphi)[k, \ell] e_{\ell} \tau_{k}+\left(|\Lambda| A_{p}(\varphi)[0,0]-A\right) I d=0 .
$$

By Theorem 2.7, the set of linear operators $\left\{e_{\ell} \tau_{k}:(k, \ell) \in \Lambda^{\circ}\right\}$ is linearly independent. Thus, $A_{p}(\varphi)[k, \ell]=0$ for every $(k, \ell) \in \Lambda^{\circ} \backslash\{(0,0)\}$ and $A=$ $|\Lambda| A_{p}(\varphi)[0,0]$. We conclude that, $A_{p}(\varphi)$ is $\Lambda^{\circ}$-sparse and the frame has the desired frame bound.

Theorem 2.2 is closely connected to the Wexler-Raz criterion. The WexlerRaz criterion checks whether a Gabor system $(\tilde{\varphi}, \Lambda)$ is a dual frame to another Gabor system, $(\varphi, \Lambda)$. Every frame $(\varphi, \Lambda)$ has at least one dual frame, $\left(S^{-1} \varphi, \Lambda\right)$, and this is known as its canonical dual frame. Theorem 2.2 is a special case of Wexler-Raz which confirms that the canonical dual frame associated with tight frames indeed satisfies the Wexler-Raz criterion. Again, a proof is not given but can be found in [35].

Theorem 2.8 (Wexler-Raz). Let $\Lambda$ be a subgroup of $(\mathbb{Z} / N \mathbb{Z}) \times(\mathbb{Z} / N \mathbb{Z})$. For Gabor systems $(\varphi, \Lambda)$ and $(\tilde{\varphi}, \Lambda)$ we have

$$
x=\sum_{(k, \ell) \in \Lambda}\left\langle x, e_{\ell} \tau_{k} \tilde{\varphi}\right\rangle e_{\ell} \tau_{k} \varphi, \quad x \in \mathbb{C}^{N},
$$

if and only if

$$
\left\langle\varphi, e_{\ell} \tau_{k} \tilde{\varphi}\right\rangle=\frac{N}{|\Lambda|} \delta_{(k, \ell),(0,0)}, \quad(k, \ell) \in \Lambda^{\circ}
$$


If $(\varphi, \Lambda)$ is a tight frame, then the cannonical dual frame to $(\varphi, \Lambda)$ is $\left(A^{-1} \varphi, \Lambda\right)$, since $S^{-1}=A^{-1} I d$. Using the Wexler-Raz criteron and using that $A=|\Lambda| A_{p}(\varphi)[0,0]$ as in Theorem 2.2, then (2.8) holds if and only if,

$$
\frac{1}{|\Lambda| A_{p}(\varphi)[0,0]}\left\langle\varphi, e_{\ell} \tau_{k} \varphi\right\rangle=\frac{N}{|\Lambda|}=\delta_{(k, \ell),(0,0)}, \quad(k, \ell) \in \Lambda^{\circ} .
$$

This can be rewritten as

$$
A_{p}(\varphi)[k, \ell]=A_{p}(\varphi)[0,0] \delta_{(k, \ell),(0,0)} \quad(k, \ell) \in \Lambda^{\circ} .
$$

This is the same condition as $A_{p}(\varphi)$ being $\Lambda^{\circ}$-sparse.

We close this section with a few operations on $\varphi$ which preservere the $\Lambda^{\circ}$ sparsity of the ambiguity function.

Proposition 2.9. Let $\varphi \in \mathbb{C}^{N} \backslash\{0\}$ and let $\Lambda \subseteq \mathbb{Z} / N \mathbb{Z} \times(\mathbb{Z} / N \mathbb{Z})^{\hat{b}}$ be a subgroup. Suppose that $A_{p}(\varphi)$ is $\Lambda^{\circ}$-sparse. Then the following are also $\Lambda^{\circ}$ - sparse:

(i) $\forall k \in \mathbb{Z} / N \mathbb{Z}, A_{p}\left(\tau_{k} \varphi\right)$

(ii) $\forall \ell \in(\mathbb{Z} / N \mathbb{Z}), A_{p}\left(e_{\ell} \varphi\right)$

(iii) $\forall c \in \mathbb{C} \backslash\{0\}, A_{p}(c \varphi)$

(iv) $A_{p}(\bar{\varphi})$.

Proof. Each part follows from direct computation which is assisted by the formula $e_{n} \tau_{m} e_{\ell} \tau_{k}=e^{2 \pi i \ell m / N} e_{\ell+n} \tau_{k+m}$ and also noting that $A_{p}(\varphi)[m, n]=$ $\left\langle\tau_{-m} \varphi, e_{n} \varphi\right\rangle$. For each $(m, n) \in \Lambda^{\circ}$ we compute:

(i) Let $k \in(\mathbb{Z} / N \mathbb{Z})$. Then,

$$
\left\langle\tau_{-m} \tau_{k} \varphi, e_{n} \tau_{k} \varphi\right\rangle=\left\langle\tau_{-m} \varphi, e_{0} \tau_{-k} e_{n} \tau_{k} \varphi\right\rangle=\left\langle\tau_{-m} \varphi, e_{n} \varphi\right\rangle=0 .
$$

(ii) Let $\ell \in(\mathbb{Z} / N \mathbb{Z})$. Then,

$$
\begin{aligned}
\left\langle\tau_{-m} e_{\ell} \varphi, e_{n} e_{\ell} \varphi\right\rangle & =\left\langle e_{-n-\ell} \tau_{-m} e_{\ell} \tau_{0} \varphi, \varphi\right\rangle=e^{-2 \pi i \ell m / N}\left\langle e_{-n} \tau_{-m} \varphi, \varphi\right\rangle \\
& =e^{-2 \pi i \ell m / N}\left\langle\tau_{-m} \varphi, e_{n} \varphi\right\rangle=0 .
\end{aligned}
$$

(iii) Let $c \in \mathbb{C} \backslash\{0\}$. Then,

$$
\left\langle\tau_{-m} c \varphi, e_{n} c \varphi\right\rangle=|c|^{2}\left\langle\tau_{-m} \varphi, e_{n} \varphi\right\rangle=0 .
$$

(iv) We compute,

$$
\begin{aligned}
\left\langle\tau_{-m} \bar{\varphi}, e_{n} \bar{\varphi}\right\rangle & =\left\langle e_{n} \varphi, \tau_{-m} \varphi\right\rangle=\left\langle\tau_{m} e_{n} \varphi, \varphi\right\rangle=e^{-2 \pi i m n / N}\left\langle e_{n} \tau_{m} \varphi, \varphi\right\rangle \\
& =e^{2 \pi i m n / N}\left\langle\tau_{m} \varphi, e_{-n} \varphi\right\rangle=0,
\end{aligned}
$$

since the product group inverse of $(m, n)$, i.e., $(-m,-n)$, is in $\Lambda^{\circ}$.

\section{CAZAC Sequences}

We give a brief exposition of the known results on CAZAC sequences, but a more detailed exposition on CAZAC sequences can be found in [5]. Although CAZAC sequences are defined in the introduction, for the sake of a self-contained exposition, we provide the definition once again. 
Definition 3.1. A sequence $\varphi \in \mathbb{C}^{N}$ is called a constant amplitude zero autocorrelation (CAZAC) sequence if the two properties:

$$
|\varphi[k]|=1, \quad \forall k \in(\mathbb{Z} / N \mathbb{Z})
$$

and

$$
\sum_{k=0}^{N-1} \varphi[k+m] \overline{\varphi[k]}=0, \quad \forall m \in(\mathbb{Z} / N \mathbb{Z}) \backslash\{0\}
$$

are satisfied.

An equivalent definition of CAZAC sequences are sequences where both $\varphi$ and $\widehat{\varphi}$ satisfiy $(\mathrm{CA})$. As such, CAZAC sequences are sometimes refered to biunimodular sequences. This idea is made clearer by Proposition 3.6. There are seven CAZAC sequences which shall be used in the examples: Chu, P4, Wiener, square-length Björck-Saffari, Milewski, and Björck sequences. We define these sequences now, but leave the analysis of their DPAFs to Section 4.

Definition 3.2. Let $\varphi \in \mathbb{C}^{N}$ be of the form

$$
\varphi[k]=e^{\pi i p[k] / N},
$$

where $p[k]$ is a polynomial. Then we can define the Chu, P4, and Wiener CAZAC sequences with,

- Chu: $p[k]=k(k-1)$ if $N$ is odd.

- P4: $p[k]=k(k-N)$ for any $N$.

- Wiener (odd length): $p[k]=s k^{2}$, if $N$ is odd and $\operatorname{gcd}(s, N)=1$.

- Wiener (even length): $p[k]=s k^{2} / 2$, if $N$ is even and $\operatorname{gcd}(s, 2 N)=1$.

All three of these sequences belong to a class of sequences known as chirp sequences. Chirp sequences are sequence whose frequency change linearly in time. They are also known as quadratic phase sequences. Some additional exposition about these sequences can be found in [9].

Definition 3.3. Let $c \in \mathbb{C}^{N}$ be CA, $v \in \mathbb{C}^{M}$ be CAZAC, and $\sigma$ be any permutation of the set $\{0,1, \cdots, N-1\}$. Then,

(i) The square length Björck-Saffari sequence, $\varphi \in \mathbb{C}^{N^{2}}$, is defined by,

$$
\forall r \in(\mathbb{Z} / N \mathbb{Z}), h \in(\mathbb{Z} / N \mathbb{Z}), \varphi[r N+h]=c[h] e^{2 \pi i r \sigma(h) / N} .
$$

(ii) The Milewski sequence, $\varphi \in \mathbb{C}^{M N^{2}}$ is defined by,

$$
\forall a \in(\mathbb{Z} / M N \mathbb{Z}), b \in(\mathbb{Z} / N \mathbb{Z}), \varphi[a N+b]=v[a] e^{2 \pi i a b / M N} .
$$

The square-length Björck-Saffari sequences were defined by Björck and Saffari as a building block to a more general class of CAZAC sequences whose lengths are not necessarily a perfect square [11]. The Milewski sequence was first defined by Milewski in [33] as a way to construct more CAZAC sequences out of already existing ones. 
Definition 3.4. Let $p$ be prime. The Legendre symbol is defined by

$$
\left(\frac{k}{p}\right)= \begin{cases}0, & \text { if } k \equiv 0 \quad \bmod p \\ 1, & \text { if } k \equiv n^{2} \quad \bmod p \text { for some } n \not \equiv 0 \bmod p \\ -1, & \text { otherwise. }\end{cases}
$$

Definition 3.5. Let $p$ be prime and let $\varphi \in \mathbb{C}^{p}$ be of the following form,

$$
\varphi[k]=e^{i \theta[k]} .
$$

We define the Björck sequence by letting $\theta[k]$ in (3.1) be as follows,

- If $p \equiv 1 \bmod 4$ then,

$$
\theta[k]=\left(\frac{k}{p}\right) \arccos \left(\frac{1}{1+\sqrt{p}}\right) .
$$

- If $p \equiv-1 \bmod 4$ then,

$$
\theta[k]= \begin{cases}\arccos \left(\frac{1-p}{1+p}\right), & \text { if }\left(\frac{k}{p}\right)=-1 \\ 0 & \text { otherwise. }\end{cases}
$$

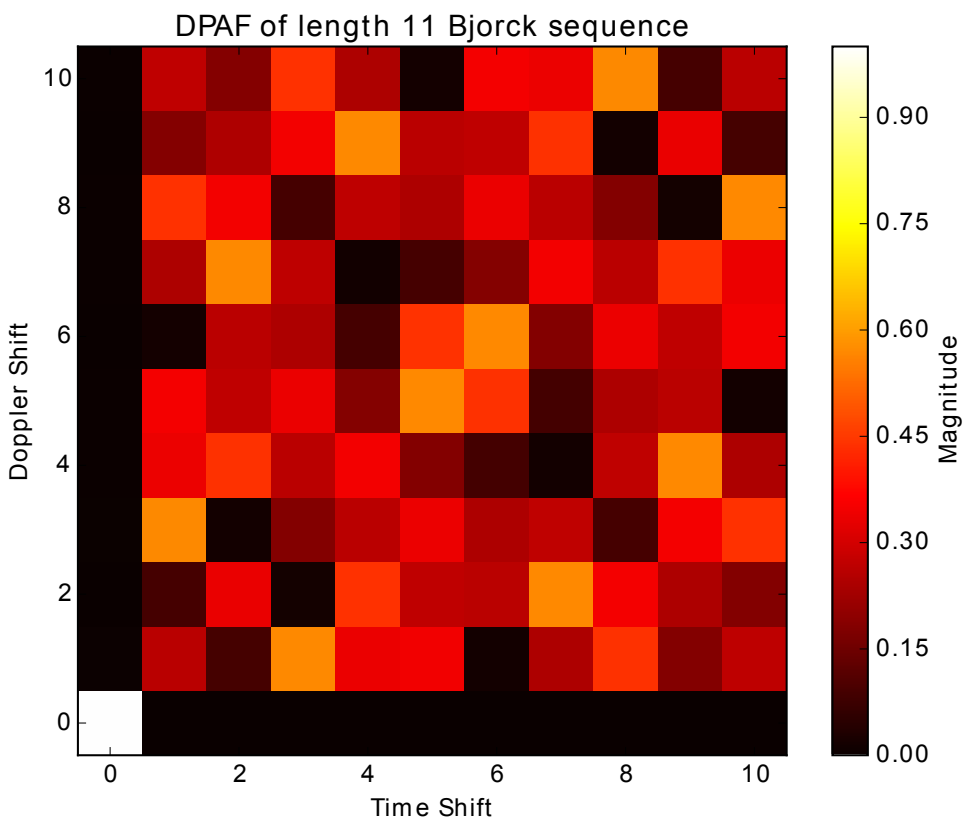

Figure 3.1. DPAF of length 11 Björck sequence.

Figure 3.1 shows that for $p=11$, the Björck sequence is indeed a CAZAC sequence. The Björck sequence is also CAZAC for any other prime length, $p$ [10]. In this particular example, one can see that the discrete periodic ambiguity 
function of the length 11 Björck sequence is almost always nonzero, except for $A_{p}(\varphi)[\cdot, 0]$ and $A_{p}(\varphi)[0, \cdot]$. This holds for any Björck sequence of any prime length, $p \geq 7$. In light of Theorem 2.2, we can see that Björck sequences are ill-suited to the construction of tight frames, despite being CAZAC. We explore this idea more in Section 5. More detailed exploration on the behavior of the DPAF of the Björck sequence can be found in [4] and [25]. For completeness, the length 11 Björck sequence is listed out below,

$$
\varphi=\left(1,1, e^{i \theta_{11}}, 1,1,1, e^{i \theta_{11}}, e^{i \theta_{11}}, e^{i \theta_{11}}, 1, e^{i \theta_{11}}\right),
$$

where $\theta_{11}=\arccos (-10 / 11)$.

Proposition 3.6. Let $\varphi \in \mathbb{C}^{N}$ and let $c \in \mathbb{C}$ be such that $|c|=1$. Then,

(i) If $\varphi$ is $C A$, then $\widehat{\varphi}$ is $Z A C$.

(ii) If $\varphi$ is $C A Z A C$, then $\widehat{\varphi}$ is also $C A Z A C$.

(iii) If $\varphi$ is $C A$, then $\widehat{\varphi}$ can have zeros.

(iv) If $\varphi$ is $C A Z A C$, then $c \varphi$ is also $C A Z A C$.

Property (iv) allows us to say two CAZAC sequences are equivalent if they are complex rotations of each other. We assume that the representative CAZAC sequence in each equivalence class is the sequence whose first entry is 1 . Given this, a natural question is as follows: For each $N$, how many CAZAC sequences of length $N$ are there? If $N$ is prime, then there are only finitely many classes [23]. On the other hand, if $N$ is composite and is not square-free, then there are infinitely many classes [11]. If $N$ is composite and square-free, it is unknown whether the number of equivalence classes is finite or infinite. Another question is, what other CAZAC sequences can we construct besides the $\mathrm{Chu}, \mathrm{P} 4$, etc.? The problem of discovering other CAZAC sequences can be transformed into two different problems in very different areas of mathematics. We present these two other equivalent problems: The first involves circulant Hadamard matrices and the second inovlves cyclic n-roots.

Definition 3.7. Let $H \in \mathbb{C}^{N \times N}$.

(i) $H$ is a Hadamard matrix if for every $(i, j),\left|H_{i, j}\right|=1$ and $H H^{*}=N I d$.

(ii) $H$ is a circulant matrix if for each $i$, the $i$-th row is a circular shift of the first row by $i-1$ entries to the right.

One can construct a circulant Hadamard matrix by making the first row a CAZAC sequence and each row after a shift of the previous row to the right. It is clear that this construction leads to a circulant matrix, and the ZAC property will guarantee that $H H^{*}=N I d$. The following theorem shows the converse, i.e. circulent Hadamard matrices give CAZAC sequences [9].

Theorem 3.8. $\varphi \in \mathbb{C}^{N \times N}$ is a CAZAC sequence if and only if the circulant matrix generated by $\varphi$ is a Hadamard matrix.

In particular, Theorem 3.8 gives a one-to-one correspondence between circulant $N \times N$ Hadamard matrices whose diagonal consists of 1 s and of CAZAC 
sequences of length $N$ whose first entry is 1 . Therefore, computing circulant Hadamard matrices is equivalent to the discovery of CAZAC sequences. There is a significant amount of research and interest in Hadamard matrices, even outside of the context of CAZACs. A catalogue of complex Hadamard matrices with relevant citations can be found online at [12].

Definition 3.9. $\left(z_{0}, z_{1}, \cdots, z_{N-1}\right) \in \mathbb{C}^{N}$ is a cyclic $N$-root if it satisfies the following system of equations,

$$
\left\{\begin{array}{l}
z_{0}+z_{1}+\cdots+z_{N-1}=0 \\
z_{0} z_{1}+z_{1} z_{2}+\cdots+z_{N-1} z_{0}=0 \\
z_{0} z_{1} z_{2}+z_{1} z_{2} z_{3}+\cdots+z_{N-1} z_{0} z_{1}=0 \\
\cdots \cdots \\
z_{0} z_{1} z_{2} \cdots z_{N-1}=1
\end{array} .\right.
$$

Let $\varphi \in \mathbb{C}^{N}$ be CAZAC with $\varphi[0]=1$. Let us emphasize the sequence nature of $\varphi \in \mathbb{C}^{N}$ and write $\varphi[k]=\varphi_{k}$. Then,

$$
\left(z_{0}, z_{1}, \cdots, z_{N-1}\right):=\left(\frac{\varphi_{1}}{\varphi_{0}}, \frac{\varphi_{2}}{\varphi_{1}}, \cdots, \frac{\varphi_{0}}{\varphi_{N-1}}\right)
$$

is a cyclic n-root. In fact, there is a one-to-one correspondence between cyclic n-roots and CAZAC sequences whose first entry is one [23]. Like with circulant Hadamard matrices, finding cyclic N-roots is equivalent to finding CAZAC sequences of length $N$. In particular, using (3.3) we can see that we can construct CAZAC sequences by the following (recursive) formula:

$$
\forall k \in\{1,2, \cdots, N-1\}, \varphi_{0}=1, \varphi_{k}=\varphi_{k-1} z_{k-1} .
$$

Cyclic $n$-roots can be used to show that the number of prime length CAZACs is finite. This was proved by Haagerup [23], but a summary, along with more results on CAZACs, can be found in [5].

\section{Discrete periodic ambiguity functions of selected sequences}

4.1. Chu Sequence. The computation of the DPAF of the Chu sequence is as follows,

$$
\begin{aligned}
A_{p}(\varphi)[m, n] & =\frac{1}{N} \sum_{k=0}^{N-1} e^{\pi i(k+m)(k+m-1) / N} e^{-\pi i k(k-1) / N} e^{-2 \pi i n k / N} \\
& =\frac{1}{N} e^{\pi i\left(m^{2}-m\right) / N} \sum_{k=0}^{N-1} e^{2 \pi i k(m-n) / N} \\
& = \begin{cases}e^{\pi i\left(m^{2}-m\right) / N}, & \text { if } m \equiv n \bmod N \\
0, & \text { otherwise }\end{cases}
\end{aligned}
$$


4.2. P4 Sequence. The computation of the DPAF of the P4 sequence is as follows,

$$
\begin{aligned}
A_{p}(\varphi)[m, n] & =\frac{1}{N} \sum_{k=0}^{N-1} e^{\pi i(k+m)(k+m-N) / N} e^{-\pi i k(k-N) / N} e^{-2 \pi i n k / N} \\
& =\frac{1}{N}(-1)^{m} e^{\pi i m^{2} / N} \sum_{k=0}^{N-1} e^{2 \pi i(m-n) k / N} \\
& = \begin{cases}(-1)^{m} e^{\pi i m^{2} / N}, & \text { if } m \equiv n \bmod N \\
0, & \text { otherwise. }\end{cases}
\end{aligned}
$$

Note that for both the Chu and P4 sequence, the DPAF is only nonzero on the diagonal $m=n$. We leverage this fact for several examples in Section 5 , and the examples use the $\mathrm{Chu}$ and $\mathrm{P} 4$ sequences interchangably.

4.3. Wiener Sequences. We start with the odd length case where $\varphi$ has the form

$$
\forall k \in(\mathbb{Z} / N \mathbb{Z}), \varphi[k]=e^{2 \pi i s k^{2} / N} .
$$

Then, the computation of the DPAF is as follows,

$$
\begin{aligned}
A_{p}(\varphi)[m, n] & =\frac{1}{N} \sum_{k=0}^{N-1} e^{2 \pi i s(k+m)^{2} / N} e^{-2 \pi i s k^{2} / N} e^{-2 \pi i n k / N} \\
& =\frac{1}{N} e^{2 \pi i s m^{2} / N} \sum_{k=0}^{N-1} e^{2 \pi i(2 s m-n) k / N} \\
& = \begin{cases}e^{2 \pi i s m^{2} / N}, & \text { if } 2 s m \equiv n \bmod N \\
0, & \text { otherwise. }\end{cases}
\end{aligned}
$$

The second case is the even length case where $\varphi$ has the form

$$
\forall k \in(\mathbb{Z} / N \mathbb{Z}), \varphi[k]=e^{\pi i s k^{2} / N} .
$$

In this case, the computation of the DPAF is as follows,

$$
\begin{aligned}
A_{p}(\varphi)[m, n] & =\frac{1}{N} \sum_{k=0}^{N-1} e^{\pi i s(k+m)^{2} / N} e^{-\pi i s k^{2} / N} e^{-2 \pi i n k / N} \\
& =e^{\pi i s m^{2} / N} \frac{1}{N} \sum_{k=0}^{N-1} e^{2 \pi i(s m-n) k / N} \\
& = \begin{cases}e^{\pi i s m^{2} / N}, & \text { if } s m \equiv n \bmod N \\
0, & \text { otherwise. }\end{cases}
\end{aligned}
$$


4.4. Square Length Björck-Saffari Sequences. Let $\omega=e^{-2 \pi i / N^{2}}$. The computation for the DPAF of the square length Björck-Saffari sequences is as follows,

$$
\begin{aligned}
& A_{p}(\varphi)[s N+t, k N+\ell] \\
& =\frac{1}{N^{2}} \sum_{r=0}^{N-1} \sum_{h=0}^{N-1} \varphi[(r+s) N+(h+t)] \overline{\varphi[r N+h]} \omega^{(k N+\ell)(r N+h)} \\
& =\frac{1}{N^{2}} \sum_{h=0}^{N-1} c[h+t] \overline{c[h]} \omega^{-N\left(s+\left\lfloor\frac{(h+t)}{N}\right\rfloor\right) \sigma(h+t)} \omega^{k h N+\ell h} \sum_{r=0}^{N-1} \omega^{-N(\sigma(h+t)-\sigma(h)-\ell) r} \\
& =\frac{1}{N} \sum_{h=0}^{N-1} c[h+t] \overline{c[h]} \omega^{-N\left(s+\left\lfloor\frac{(h+t)}{N}\right\rfloor\right) \sigma(h+t)} \omega^{k h N+\ell h}
\end{aligned}
$$

if $\sigma(h+t)-\sigma(h)-\ell \equiv 0 \bmod N$, and 0 otherwise. In particular, if $\sigma(h)=h$ for all $h$, then the above condition reduces to $t \equiv \ell \bmod N$.

4.5. Milewski sequences. We shall write out the DPAF of the Milewski sequence without computation, but the computation can be found in [6]. For the DPAF of the Milewski sequence, $A_{p}(\varphi)$ we have that $N A_{p}(\varphi)[k N+\ell, s]$ is computed by

$$
\left\{\begin{array}{lll}
0, & \text { if } m \neq \equiv n & \bmod N \\
\sum_{j=0}^{N-1} e^{2 \pi i\left(k+\left\lfloor\frac{j+\ell}{N}\right\rfloor-j s\right) / M N} A_{p}(v)\left[k+\left\lfloor\frac{j+\ell}{N}\right\rfloor, \frac{s-\ell}{N}\right], & \text { if } m \equiv n & \bmod N
\end{array}\right.
$$

\section{Examples of tight Gabor frames generated by $\Lambda^{\circ}$-sparsity}

For most of the examples, we will use the following formulation of subgroup $\Lambda$ : Let $K=\langle a\rangle$ and $L=\langle b\rangle$ where $N=a b N^{\prime}$ and $\operatorname{gcd}(a, b)=1$. That is, $K$ is the subgroup of $(\mathbb{Z} / N \mathbb{Z})$ generated by $a:\left\{0, a, \cdots,\left(b N^{\prime}-1\right) a\right\}$ and $L$ is the subgroup of $(\mathbb{Z} / N \mathbb{Z})^{-}$generated by $b$ : $\left\{0, b, \cdots,\left(a N^{\prime}-1\right) b\right\}$. Let $\Lambda=K \times L$. We first shall compute the adjoint subgroup of $\Lambda=K \times L$. This requires us to compute which time-frequency translates $e_{n} \tau_{m}$ commute with every timefrequency translate $e_{\ell} \tau_{k}$ with $(k, \ell) \in K \times L$. To that end, let $e_{\ell} \tau_{k}$ with $(k, \ell) \in$ $K \times L$, and $e_{n} \tau_{m}$ be two time-frequency translates. We noted in Proposition 2.9 that $e_{n} \tau_{m} e_{\ell} \tau_{k}=e^{-2 \pi i \ell m / N} e_{\ell+n} \tau_{k+m}$. Switching $k$ and $m$, as well as $\ell$ and $n$, we have $e_{\ell} \tau_{k} e_{n} \tau_{m}=e^{-2 \pi i k n / N} e_{\ell+n} \tau_{k+m}$. Thus, $(m, n) \in \Lambda^{\circ}$ if and only if

$$
\ell m \equiv k n \quad \bmod N, \quad \forall(k, \ell) \in \Lambda \text {. }
$$

Since $k \in K$ and $\ell \in L$ we can write $k=k^{\prime} a$ and $\ell=\ell^{\prime} b$ for some $k^{\prime} \in\left(\mathbb{Z} / b N^{\prime} \mathbb{Z}\right)$ and $\ell^{\prime} \in\left(\mathbb{Z} / a N^{\prime} \mathbb{Z}\right)$. Using this in (5.1) gives a new condition

$$
\ell^{\prime} b m \equiv k^{\prime} \text { an } \bmod N, \forall\left(k^{\prime}, \ell^{\prime}\right) \in\left(\mathbb{Z} / b N^{\prime} \mathbb{Z}\right) \times\left(\mathbb{Z} / a N^{\prime} \mathbb{Z}\right) .
$$


Lemma 5.1. Let $N=a b N^{\prime}$ where $\operatorname{gcd}(a, b)=1 .(m, n)$ is a solution to (5.2) if and only if $m$ is a multiple of $N^{\prime} a$ and $n$ is a multiple of $N^{\prime} b$. In particular, if $\Lambda=K \times L$ where $K=\langle a\rangle$ and $L=\langle b\rangle$, then $\Lambda^{\circ}=N^{\prime} K \times N^{\prime} L=\left\langle N^{\prime} a\right\rangle \times\left\langle N^{\prime} b\right\rangle$.

Proof. First note that if $m=r N^{\prime} a$ and $n=s N^{\prime} b$ for some $r \in(\mathbb{Z} / b \mathbb{Z})$ and $s \in(\mathbb{Z} / a \mathbb{Z})$ then the left hand side of $(5.2)$ becomes

$$
\ell^{\prime} b\left(r N^{\prime} a\right) \equiv \ell^{\prime} r\left(a b N^{\prime}\right) \equiv 0 \bmod N .
$$

The right hand side of (5.2) becomes

$$
k^{\prime} a\left(s N^{\prime} b\right) \equiv k^{\prime} s\left(a b N^{\prime}\right) \equiv 0 \bmod N .
$$

Since (5.3) and (5.4) are equal we have that $N^{\prime} K \times N^{\prime} L \subseteq \Lambda^{\circ}$. Conversely, note that since $\operatorname{gcd}(a, b)=1, m$ must be a multiple of $a$, and $n$ must be a multiple of $b$. In other words, $\Lambda^{\circ} \subseteq K \times L$. Now, suppose $m=r a$ and $n=s b$. Then, condition (5.2) becomes

$$
\ell^{\prime} r a b \equiv k^{\prime} s a b \bmod N, \forall\left(k^{\prime}, \ell^{\prime}\right) \in\left(\mathbb{Z} / b N^{\prime} \mathbb{Z}\right) \times\left(\mathbb{Z} / a N^{\prime} \mathbb{Z}\right) .
$$

If $N^{\prime}=1$, the above is always true since $N=a b N^{\prime}=a b$ and thus $\Lambda^{\circ}=$ $N^{\prime} K \times N^{\prime} L=K \times L$. Assume that $N^{\prime}>1$ and without loss of generality, assume $N^{\prime} \nmid r$. Then, choose $\ell^{\prime}=1$ and $k^{\prime}=0$. We now have that the right hand side is 0 while the left hand side cannot be a multiple of $N$. Thus, (5.5) cannot hold, $N^{\prime} \mid r$ and $N^{\prime} \mid s$ is necessary, and we get $\Lambda^{\circ} \subseteq N^{\prime} K \times N^{\prime} L$.

The first example uses the $K \times L$ setup and applies it to the Chu and P4 sequence. As seen in Section 4.1 and Section 4.2, if $\varphi$ is the $\mathrm{Chu}$ or $\mathrm{P} 4$ sequence, $A_{p}(\varphi)[m, n]$ only has nonzero entries along the diagonal $m=n$. We will leverage this fact for an easy proof of Proposition 5.2. It should also be noted that some slight modifications can be made to easily extend Proposition 5.2 to Wiener sequences.

Proposition 5.2. Let $\varphi \in \mathbb{C}^{N}$ be either the Chu or P4 sequence and let $K=$ $\langle a\rangle$ and $L=\langle b\rangle$ with $\operatorname{gcd}(a, b)=1$ and $N=a b N^{\prime}$. Then, the Gabor system $(\varphi, K \times L)$ is a tight Gabor frame with frame bound $N N^{\prime}$.

Proof. Using Lemma 5.1, we have that $(m, n) \in(K \times L)^{\circ}$ if and only if $m=$ $r a N^{\prime}$ and $n=s b N^{\prime}$ for some $r \in\left(\mathbb{Z} / b N^{\prime} \mathbb{Z}\right)$ and $s \in\left(\mathbb{Z} / a N^{\prime} \mathbb{Z}\right)$. However, $A_{p}(\varphi)[m, n] \neq 0$ if and only if $m \equiv n \bmod N$, i.e. $m, n \in N^{\prime} K \cap N^{\prime} L$. This intersection is generated by $\operatorname{lcm}\left(N^{\prime} a, N^{\prime} b\right)=a b N^{\prime}=N$. Thus, for $(m, n) \in$ $(K \times L)^{\circ}, A_{p}(\varphi)[m, n] \neq 0$ if and only if $m=n=0$. By Theorem 2.2, we have that $(\varphi, K \times L)$ is a tight frame with frame bound $|\Lambda|=N N^{\prime}$.

Note that other subgroups besides the $K \times L$ type subgroups can be used in Proposition 5.2 to obtain the same result. The proof only required that for every $(m, n) \in \Lambda^{\circ}, m \not \equiv n \bmod N$ unless $(m, n)=(0,0)$. We provide an example illustrating this idea, Proposition 5.3.

Proposition 5.3. Let $\varphi \in \mathbb{C}^{N}$ be either the Chu or P4 sequence and let $\Lambda=$ $\langle(a, b)\rangle$ where $a \neq b$ and $\operatorname{gcd}(a, N)=\operatorname{gcd}(b, N)=1$. Then, the Gabor system $(\varphi, \Lambda)$ is a tight Gabor frame with frame bound $N$. 
Proof. Suppose $\operatorname{gcd}(a, b)=d$. Let $a=d a^{\prime}$ and $b=d b^{\prime}$. Since $\operatorname{gcd}(a, N)=1$ and $\operatorname{gcd}(b, N)=1$, the order of $\Lambda$ is $N$ and is the same subgroup as the one generated by $\left(a^{\prime}, b^{\prime}\right)$. Thus, without loss of generality, we can assume that $\operatorname{gcd}(a, b)=1$. Then, by (5.1), we know that $(m, n) \in \Lambda^{\circ}$ if and only if

$$
k n \equiv \ell m \quad \bmod N
$$

which can be rewritten as

$$
\text { san } \equiv s b m \quad \bmod N .
$$

Letting $s=1$, we see that $a n \equiv b m \bmod N$ is a necessary condition and since $\operatorname{gcd}(a, b)=1$ we need that $n$ is a multiple of $b$ and $m$ is a multiple of $a$. It is clear that multiples of $(a, b)$ also work for $s \neq 1$ and so we have that $\Lambda^{\circ}=\Lambda$. Since $\operatorname{gcd}(a, b)=1$, we have that $m=n$ if and only if $m=n=0$. Thus, $A_{p}(\varphi)[m, n]=0$ for every $(m, n) \in \Lambda^{\circ}$ except at $(0,0)$. Since $|\Lambda|=N$, we have that $(\varphi, \Lambda)$ is a tight frame with frame bound $N$.

Next, we examine the square length Björck-Saffari sequence. From Section 4.4, the DPAF of the square length Björck-Saffari sequence is still sparse, and the nonzero entries have regular structure when we use the identity permutation $\sigma(m)=m$. We leverage this fact to prove Proposition 5.4 and apply the same techniques as in Proposition 5.2.

Proposition 5.4. Let $c \in \mathbb{C}^{N}$ and let $\varphi \in \mathbb{C}^{N^{2}}$ be the square length BjörckSaffari sequence generated by $c$ and $\sigma(h)=h$. Let $K=\langle a\rangle$ and $L=\langle b\rangle$ with $\operatorname{gcd}(a, b)=1$ and $N^{2}=a b N^{\prime}$. Then, the Gabor system $(\varphi, K \times L)$ is a tight Gabor frame with frame bound $N^{2} N^{\prime}$.

Proof. Using Lemma 5.1, $\sigma(h)=h$, and the results of Section 4.4, if $\left(r N^{\prime} a, s N^{\prime} b\right) \in$ $N^{\prime} K \times N^{\prime} L$, then $A_{p}(\varphi)\left[r N^{\prime} a, s N^{\prime} b\right] \neq 0$ only if

$$
r N^{\prime} a \equiv s N^{\prime} b \quad \bmod N
$$

for some $r, s \in(\mathbb{Z} / N \mathbb{Z})$. From the proof of Proposition 5.2, we know that $N^{\prime} K \cap N^{\prime} L$ is generated by $\operatorname{lcm}\left(a N^{\prime}, b N^{\prime}\right)$. We have that $\operatorname{lcm}\left(N^{\prime} a, N^{\prime} b\right)=N^{\prime}$ $\operatorname{lcm}(a, b)=a b N^{\prime}=N^{2} \equiv 0 \bmod N$. Moreover, if $A_{p}(\varphi)\left[r N^{\prime} a, s N^{\prime} b\right] \neq 0$, then $r=s=0$. By Theorem 2.2, we have that $(\varphi, K \times L)$ is a tight Gabor frame, and the frame bound is $|\Lambda|=N^{2} N^{\prime}$.

The next example utilizes Theorem 2.2, but has the opposite theme: the discrete periodic amibguity function is mostly nonzero except for the "right" spots. That is, $A_{p}(\varphi)[m, n] \neq 0$ for nearly every $(m, n) \notin \Lambda^{\circ}$ but $A_{p}(\varphi)$ will still be $\Lambda^{\circ}$-sparse.

Proposition 5.5. Let $u \in \mathbb{C}^{M}$ be $C A Z A C, v \in \mathbb{C}^{N}$ be $C A$, and $\varphi \in \mathbb{C}^{M N}$ be defined by

$$
\varphi=u \otimes v
$$

where $\otimes$ is the Kronecker product. Assume $\operatorname{gcd}(M, N)=1$ and let $\Lambda=\langle M\rangle \times$ $\langle N\rangle$. Then, $(\varphi, \Lambda)$ is a tight frame with frame bound $M N$. 
Proof. We can write the $(r N+s)$-th term of $\varphi$ as

$$
\varphi[r N+s]=u[r] v[s]
$$

where $r \in(\mathbb{Z} / M \mathbb{Z})$ and $s \in(\mathbb{Z} / N \mathbb{Z})$. We now compute the DPAF,

$$
\begin{aligned}
& A_{p}(\varphi)[r N+s, \ell] \\
& =\frac{1}{M N} \sum_{j=0}^{M-1} \sum_{k=0}^{N-1} u\left[j+r+\left\lfloor\frac{s+k}{N}\right\rfloor\right] v[s+k] \overline{u[j] v[k]} e^{-2 \pi i(j N+k) \ell / M N} \\
& =\frac{1}{N} \sum_{k=0}^{N-1} v[s+k] \overline{v[k]} e^{-2 \pi i k \ell / M N} \frac{1}{M} \sum_{j=0}^{M-1} u\left[j+r+\left\lfloor\frac{s+k}{N}\right\rfloor\right] \overline{u[j]} e^{-2 \pi i j \ell / M} \\
& =\frac{1}{N} \sum_{k=0}^{N-1} v[s+k] \overline{v[k]} e^{-2 \pi i k \ell / M N} A_{p}(u)\left[r+\left\lfloor\frac{s+k}{N}\right\rfloor, \ell\right] .
\end{aligned}
$$

By Lemma 5.1 we have that $\Lambda^{\circ}=\Lambda$. Using (5.7), we can see that for $(r N, \ell M) \in$ $\Lambda^{\circ} \backslash\{(0,0)\}$ we have

$$
\begin{aligned}
A_{p}(\varphi)[r N, \ell M] & =\frac{1}{N} \sum_{k=0}^{N-1} v[k] \overline{v[k]} e^{-2 \pi i k \ell / N} A_{p}(u)[r, \ell M] \\
& =\frac{A_{p}(u)[r, 0]}{N} \sum_{k=0}^{N-1}|v[k]|^{2} e^{-2 \pi i k \ell / N}=0
\end{aligned}
$$

since one of $r$ or $\ell$ is nonzero. Indeed, if $r$ is nonzero, then since $u$ has zero autocorrelation, we have a multiplier of zero outside of the sum. On the other hand, if $r=0$ but $\ell \neq 0$, then the sum will add up to zero since $|v[k]|^{2}=1$ for every $k$. We now conclude by Theorem 2.2 that $(\varphi, \Lambda)$ is a tight frame with frame bound $M N$.

Although $A_{p}(\varphi)$ is $\Lambda^{\circ}$-sparse, (5.7) implies that most of the entries for $A_{p}(\varphi)$ are nonzero. This is illustrated by Figure 5.1. In Figure 5.1, $u$ is the Björck sequence which is defined in Section 3.

Proposition 5.6. Let $\varphi \in \mathbb{C}^{M N^{2}}$ be a Milewski sequence where the generating $v \in \mathbb{C}^{M}$ is either the Chu or P4 sequence, and let $a, b, j, N^{\prime}$ be such that $\operatorname{gcd}(a, b)=1, j \mid M N, N^{\prime}=j N$, and $a b N^{\prime}=M N^{2}$. Let $\Lambda=K \times L$ where $K=\langle a\rangle$, and $L=\langle b\rangle$. Then, $(\varphi, \Lambda)$ is a tight frame with frame bound $j M N^{3}$.

Proof. By Lemma 5.1, we have that $\Lambda^{\circ}=N^{\prime} K \times N^{\prime} L$. In particular, for every $(m, n) \in \Lambda^{\circ}$ we have that $m \equiv n \equiv 0 \bmod N$. Thus, using the second line of (4.1), for $(m, n) \in \Lambda^{\circ}$ we can write $A_{p}(\varphi)[m, n]$ in the form

$$
N A_{p}(\varphi)[m, n]=\sum_{j=0}^{N-1} e^{\theta[j]} A_{p}(v)\left[m^{\prime}, n^{\prime}\right]
$$




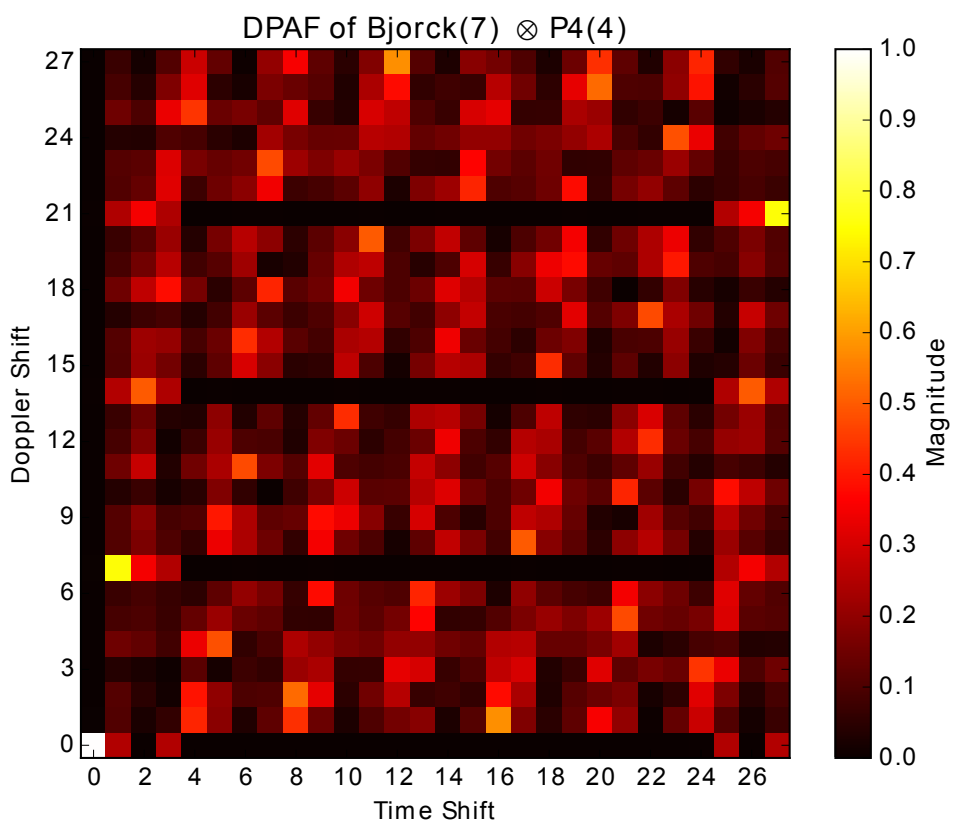

Figure 5.1. DPAF of $\varphi=u \otimes v$ where $u$ is the length 7 Björck sequence and $v$ is the length $4 \mathrm{P} 4$ sequence.

where $m^{\prime}=m / N$ and $n^{\prime}=n / N$. Since $v$ is the Chu or $\mathrm{P} 4$ sequence, we have that $A_{p}(v)\left[m^{\prime}, n^{\prime}\right] \neq 0$ if and only if $m^{\prime} \equiv n^{\prime} \bmod M$. Furthermore, $\Lambda^{\circ}=\left\langle a N^{\prime}\right\rangle \times$ $\left\langle b N^{\prime}\right\rangle=\langle a j N\rangle \times\langle b j N\rangle$ and so we have that $m^{\prime} \in\langle a j\rangle$ and $n^{\prime} \in\langle b j\rangle$. Thus, we have that $m^{\prime} \equiv n^{\prime} \bmod M$ if and only if $m^{\prime}, n^{\prime} \in\langle a j\rangle \cap\langle b j\rangle=\langle\operatorname{lcm}(a j, b j)\rangle$. Since $\operatorname{gcd}(a, b)=1, \operatorname{lcm}(a j, b j)=a b j=M N$ since $a b j N=a b N^{\prime}=M N^{2}$. We want to view $\langle a j\rangle$ and $\langle b j\rangle$ as being subgroups of $(\mathbb{Z} / M N \mathbb{Z})$, thus we have that $m^{\prime} \equiv n^{\prime} \bmod M N$ if and only if $m^{\prime} \equiv n^{\prime} \equiv 0 \bmod M N$. Therefore, if $(m, n) \in \Lambda^{\circ}$, then $A_{p}(\varphi)=0$ except for $(m, n)=(0,0)$. By Theorem 2.2, we conclude that $(\varphi, \Lambda)$ is a tight frame with frame bound $j M N^{3}$.

\section{Gram matrices in terms of the discrete periodic ambiguity function}

The general framework for the last two sections is as follows: First, we explicitly compute the entries of the Gram matrix by using the discrete periodic ambiguity function. Then, we show that the first $N$ columns or rows have disjoint supports. Last, we show that every other column or row is a constant multiple of one of the first $N$ rows or columns, and show that there is only one nonzero eigenvalue. This allows us to conclude that the Gabor system is indeed a tight frame. This section is devoted to the first step, the computation of the Gram matrix for the $\mathrm{Chu}, \mathrm{P} 4$, and Wiener sequences. 
6.1. Gram Matrix of Gabor Systems. In this subsection, we show that each entry of the Gram matrix of a Gabor system can be written in terms of the discrete periodic ambiguity function of the $\varphi \in \mathbb{C}^{N} \backslash\{0\}$ which generates the system. To start, we will define the Gram matrix and the theorem which will establish the main tool for determining when frames are tight via its Gram matrix.

Definition 6.1. Let $\mathcal{H}$ be a Hilbert space and let $\mathcal{F}$ be a frame for $\mathcal{H}$. Let $F$ and $F^{*}$ be the analysis operator and synthesis operator of $\mathcal{F}$, respectively, as defined in Definition 2.5. The Gram operator, $G$, is defined by $G=F^{*} F$.

If we write $\mathcal{F}=\left\{v_{i}\right\}_{i=1}^{M}$ (with $M \geq N$ ) and explicitly write out $G$, it can be shown that the Gram operator can be written in matrix form by writing the $(i, j)$-th entry as $G_{i j}=\left\langle v_{i}, v_{j}\right\rangle$. We use this formula in most of the computations involving the Gram matrix. We also use the following theorem in Section 7.

Theorem 6.2. Let $\mathcal{H}$ be an $N$-dimensional Hilbert space and let $\mathcal{F}=\left\{v_{i}\right\}_{i=1}^{M}$ be a frame for $\mathcal{H} . \mathcal{F}$ is a tight frame if and only if $\operatorname{rank}(G)=N$ and $G$ has a single eigenvalue.

For all that follows let $\varphi \in \mathbb{C}^{N} \backslash\{0\}$ and let us write out the Gabor system as $\mathcal{F}=\left\{e_{\ell_{m}} \tau_{k_{m}} \varphi: m \in 0, \cdots, M\right\}$. Then, we can compute the $(m, n)$-th entry of the Gram matrix:

$$
\begin{aligned}
G_{m, n} & =\left\langle e_{\ell_{m}} \tau_{k_{m}} \varphi, \overline{e_{\ell_{n}} \tau_{k_{n}} \varphi}\right\rangle=\sum_{j=0}^{N} e^{2 \pi i \ell_{m} j / N} \varphi\left[j-k_{m}\right] e^{-2 \pi i \ell_{n} j / N \overline{\varphi\left[j-k_{n}\right]}} \\
& =\sum_{j=0}^{N} e^{-2 \pi i\left(\ell_{n}-\ell_{m}\right) j / N} \varphi\left[j-k_{m}\right] \overline{\varphi\left[j-k_{n}\right]} \\
& =\sum_{j=0}^{N} e^{-2 \pi i\left(\ell_{n}-\ell_{m}\right)\left(j-k_{n}+k_{n}\right) / N} \varphi\left[j-k_{n}+\left(k_{n}-k_{m}\right)\right] \overline{\varphi\left[j-k_{n}\right]} \\
& =e^{-2 \pi i k_{n}\left(\ell_{n}-\ell_{m}\right) / N} \sum_{j=0}^{N} \varphi\left[j+\left(k_{n}-k_{m}\right)\right] \overline{\varphi[j]} e^{-2 \pi i\left(\ell_{n}-\ell_{m}\right) j / N} \\
& =N e^{-2 \pi i k_{n}\left(\ell_{n}-\ell_{m}\right) / N} A_{p}(\varphi)\left[k_{n}-k_{m}, \ell_{n}-\ell_{m}\right]
\end{aligned}
$$

6.2. Gram Matrices of some CAZAC sequences. In this subsection, we apply the results of Section 4 and Section 6.1 to explicitly write down the Gabor matrix of the $\mathrm{Chu}, \mathrm{P} 4$, and Wiener CAZAC sequences. For convenience, we define $r_{m n}: \equiv\left(k_{n}-k_{m}\right) \equiv\left(\ell_{n}-\ell_{m}\right)$. Using this notation, we get the following Gram matrices:

\section{Chu sequence:}

$$
G_{m n}= \begin{cases}N e^{-2 \pi i\left[k_{n} r_{m n}-\frac{1}{2}\left(r_{m n}^{2}-r_{m n}\right)\right] / N}, & \text { if }\left(\ell_{n}-\ell_{m}\right) \equiv\left(k_{n}-k_{m}\right) \bmod N \\ 0, & \text { otherwise }\end{cases}
$$




\section{P4 Sequence:}

$$
G_{m n}= \begin{cases}N(-1)^{r_{m n}} e^{-2 \pi i\left[k_{n} r_{m n}-\frac{1}{2} r_{m n}^{2}\right] / N}, & \text { if }\left(k_{n}-k_{m}\right) \equiv\left(\ell_{n}-\ell_{m}\right) \bmod N \\ 0, & \text { otherwise }\end{cases}
$$

Odd Length Wiener Sequence:

$$
G_{m n}= \begin{cases}N e^{-4 \pi i s k_{n} r_{m n} / N} e^{2 \pi i s r_{m n}^{2} / N}, & \text { if } 2 s\left(k_{n}-k_{m}\right) \equiv\left(\ell_{n}-\ell_{m}\right) \quad \bmod N \\ 0, & \text { otherwise. }\end{cases}
$$

\section{Even Length Wiener Sequence:}

$$
G_{m n}= \begin{cases}N e^{-2 \pi i s k_{n} r_{m n} / N} e^{\pi i s m^{2} / N}, & \text { if } s\left(k_{n}-k_{m}\right) \equiv\left(\ell_{n}-\ell_{m}\right) \quad \bmod N \\ 0, & \text { otherwise. }\end{cases}
$$

\section{Gram matrix method}

We now apply the method outlined in Section 6 to the Chu and P4 sequence. We treat these two cases simultaneously since both have the property that $A_{p}(\varphi)[m, n]=0$ if $m \neq n$, and are nonzero when $m=n$. The computation in Lemma 7.3 is different for the $\mathrm{P} 4$ case, but the same ideas can be used achieve the same result. The Wiener case has direct analogues of the results in the Chu and $\mathrm{P} 4$ cases, and we will only highlight the key differences in the proofs instead of reiterating the details. Before proceeding, we need a useful fact about the Gram matrix which can easily be derived from the singular value decomposition of the analysis operator.

Lemma 7.1. Let $F$ be an $m \times n$ complex-valued matrix and let $G:=F F^{*}$. Then, $\operatorname{rank}(G)=\operatorname{rank}(F)$.

Proof. First, we write $F$ in terms of its SVD: $F=U D V^{*}$ where $D$ is an $m \times n$ rectangular diagonal matrix and $U$ and $V$ are $m \times m$ and $n \times n$ unitary matrices, respectively. Note that $G=F F^{*}=U D V^{*} V D^{*} U^{*}=U D D^{*} U^{*}=U D^{2} U^{*}$. In particular, $D_{i j}^{2}=\left|D_{i j}\right|^{2}$ and from this we conclude $\operatorname{rank}(G)=\operatorname{rank}(F)$.

For the following propostions, we shall use the following arrangement for the Gram matrix. Let $(\varphi, K \times L)$ be a Gabor system in $\mathbb{C}^{N}$. We shall iterate by modulation first, then iterate by translations. In other words, the analysis operator, $F$, will be a $|K||L| \times N$ matrix where the $m$-th row is given by $m=$ $r|L|+s, r \in(\mathbb{Z} /|K| \mathbb{Z}), s \in(\mathbb{Z} /|L| \mathbb{Z})$, and the $m=r|L|+s$-th row corresponds to $e_{\ell_{s}} \tau_{k_{r}} \varphi$.

Proposition 7.2. Let $N=a b$ with $\operatorname{gcd}(a, b)=1$ and let $\varphi \in \mathbb{C}^{N}$ be either the Chu or P4 sequence. Let $K=\langle a\rangle$ and $L=\langle b\rangle$. Then, the Gabor system $(\varphi, K \times L)$ is a tight frame with frame bound $N$.

Proof. By construction, $|K|=b,|L|=a$ and so the Gabor system $(\varphi, K \times L)$ has $a b=N$ vectors. Since $\operatorname{lcm}(a, b)=N$, we have that $K \cap L=\{0\}$. From Section 6.2 , we have that $G_{m, n} \neq 0$ if and only if $\left(\ell_{n}-\ell_{m}\right)=\left(k_{n}-k_{m}\right)$. By 
design of $K$ and $L$, we have that $\left(\ell_{n}-\ell_{m}\right)=j b$ and $\left(k_{n}-k_{m}\right)=\tilde{j} a$ for some $j, \tilde{j}$. In particular, $\left(k_{n}-k_{m}\right) \in K$ and $\left(\ell_{n}-\ell_{m}\right) \in L$, and can only be equal if both belong to $K \cap L$. Thus, $G_{m, n} \neq 0$ if and only if $\left(\ell_{n}-\ell_{m}\right)=\left(k_{n}-k_{m}\right)=0$, i.e. $j=\tilde{j}=0$. We conclude that the nonzero entries lie only on the diagonal of $G$. Using formulas (6.1) and (6.2), we see that for each $n \in(\mathbb{Z} / N \mathbb{Z}), G_{n, n}=N$ and $G=N I d_{N}$. Thus, the Gabor system $(\varphi, K \times L)$ is a tight frame with frame bound $N$.

Lemma 7.3. Let $\varphi \in \mathbb{C}^{N}$ be either the Chu or P4 sequence and let $N=a b N^{\prime}$ where $\operatorname{gcd}(a, b)=1$. Suppose $G$ is the Gram matrix generated by the Gabor system $(\varphi, K \times L)$ where $K=\langle a\rangle$ and $L=\langle b\rangle$. Then, there exist functions $f, g: \mathbb{N} \cup\{0\} \rightarrow \mathbb{C}$ such that $G_{m n} / N=f(n) g(m)$ wherever $G_{m n} \neq 0$.

Proof. We will only cover the case of the Chu sequence. The case of the P4 sequence follows by replacing $e^{-\pi i r_{m n} / N}$ with $(-1)^{r_{m n}}$ and carrying out the same computations. If $G_{m n} \neq 0$, then we have

$$
G_{m n} / N=e^{-2 \pi i k_{n} r_{m n} / N} e^{-\pi i r_{m n} / N} e^{\pi i r_{m n}^{2} / N}
$$

where

$$
r_{m n}=k_{n}-k_{m}=\ell_{n}-\ell_{m}=j a b .
$$

Note that $k_{n}=c_{n} a b+d_{n} a$ and $k_{m}=c_{m} a b+d_{m} a$ where $c_{n}, c_{m} \in\left(\mathbb{Z} / N^{\prime} \mathbb{Z}\right), d_{n}, d_{m} \in$ $(\mathbb{Z} / b \mathbb{Z})$. However, by $(7.2), j=c_{n}-c_{m}$ and $d_{n}=d_{m}$. Putting this back into (7.1) and using $\omega=e^{2 \pi i / N}$ and $\zeta=e^{2 \pi i / N^{\prime}}$, we have

$$
\begin{aligned}
G_{m n} / N & =\omega^{-\left(c_{n} a b+d_{n} a\right)\left(c_{n}-c_{m}\right) a b} \omega^{-\left(c_{n}-c_{m}\right) a b / 2} \omega^{\left(c_{n}-c_{m}\right)^{2} a^{2} b^{2} / 2} \\
& =\zeta^{-c_{n} a b\left(c_{n}-c_{m}\right)} \zeta^{-d_{n} a\left(c_{n}-c_{m}\right)} \zeta^{-\left(c_{n}-c_{m}\right) / 2} \zeta^{a b\left(c_{n}^{2}-2 c_{n} c_{m}+c_{m}^{2}\right) / 2} \\
& =\zeta^{-c_{n}^{2} a b} \zeta^{c_{n} c_{m} a b} \zeta^{-d_{n} a\left(c_{n}-c_{m}\right)} \zeta^{-\left(c_{n}-c_{m}\right) / 2} \zeta^{a b\left(c_{n}^{2}+c_{m}^{2}\right) / 2} \zeta^{-a b c_{n} c_{m}} \\
& =\zeta^{-c_{n}^{2} a b / 2} \zeta^{-d_{n} c_{n} a} \zeta^{-c_{n} / 2} \zeta^{c_{m}^{2} a b / 2} \zeta^{d_{m} c_{m} a} \zeta^{c_{m} / 2} .
\end{aligned}
$$

Here we have used the crucial fact that $d_{n}=d_{m}$. Thus, we can write nonzero entries of $G_{m n}$ as $f(n) g(m)$ where

$$
f(n)=\zeta^{-c_{n}^{2} a b / 2} \zeta^{-d_{n} c_{n} a} \zeta^{-c_{n} / 2}
$$

and

$$
g(m)=\zeta^{\pi i c_{m}^{2} a b / 2} \zeta^{d_{m} c_{m} a} \zeta^{c_{m} / 2}
$$

Lemma 7.4. Let $\varphi \in \mathbb{C}^{N}$ be either the Chu or P4 sequence and let $N=a b N^{\prime}$ where $\operatorname{gcd}(a, b)=1$. Suppose $G$ is the Gram matrix generated by the Gabor system $(\varphi, K \times L)$ where $K=\langle a\rangle$ and $L=\langle b\rangle$. Then, the support of the rows (or columns) of $G$ either completely coincide or are completely disjoint.

Proof. Let us denote the $m$-th row of $G$ as $g_{m}$, and all indices are implictly taken modulo $N$. We shall show that if there is at least one index $n \in(\mathbb{Z} / N \mathbb{Z})$ such that $g_{m}[n]=g_{m^{\prime}}[n] \neq 0$, and $m \neq m^{\prime}$, then $\operatorname{supp}\left(g_{m}\right)=\operatorname{supp}\left(g_{m^{\prime}}\right)$. Suppose 
that $g_{m}[n]=g_{m^{\prime}}[n] \neq 0$. Then, $k_{n}-k_{m}=\ell_{n}-\ell_{m}=j a b$ and $k_{n}-k_{m^{\prime}}=$ $\ell_{n}-\ell_{m^{\prime}}=j^{\prime} a b$. We can rearrange these two equations to obtain the following

$$
k_{m}=k_{n}-j a b
$$

and

$$
k_{n}=k_{m^{\prime}}+j^{\prime} a b .
$$

Now suppose there is another $n^{\prime} \in(\mathbb{Z} / N \mathbb{Z})$ where $g_{m}\left[n^{\prime}\right] \neq 0$. Then, we have

$$
k_{n^{\prime}}-k_{m}=\tilde{j} a b \text {. }
$$

Substiuting (7.3) and (7.4) into (7.5), we have

$$
\tilde{j} a b=k_{n^{\prime}}-k_{m}=k_{n^{\prime}}-k_{n}+j a b=k_{n^{\prime}}-k_{m^{\prime}}-j^{\prime} a b+j a b,
$$

which can be rearranged to obtain

$$
\left(j^{\prime}+\tilde{j}-j\right) a b=k_{n^{\prime}}-k_{m^{\prime}} .
$$

Note that $\left(j^{\prime}+\tilde{j}-j\right) \in(\mathbb{Z} / N \mathbb{Z})$ and that these same computations can be done replacing $k$ with $\ell$. Thus, $g_{m^{\prime}}\left[n^{\prime}\right] \neq 0$ as well and the result is proved.

Remark 7.5. Let $\operatorname{gcd}(a, b)=1, N=a b N^{\prime}, K=\langle a\rangle, L=\langle b\rangle, \varphi \in \mathbb{C}^{N}$ be the $\mathrm{Chu}$ and $\mathrm{P} 4$ sequence, and consider the system $(\varphi, K \times L)$. In light of Lemma 7.3 and Lemma 7.4, if two rows of the Gram matrix $G$ have supports which coincide, they must be constant multiples of each other, where this multiple has modulus 1. Indeed, if $g_{m}$ and $g_{m^{\prime}}$ have coinciding supports then for each $n$ where they are nonzero we have

$$
\frac{G_{m n}}{G_{m^{\prime} n}}=\frac{N f(n) g(m)}{N f(n) g\left(m^{\prime}\right)}=\frac{g(m)}{g\left(m^{\prime}\right)} .
$$

This also gives us a formula for finding the constant multiple, should we desire it. This idea is illustrated with Figure 7.1, where two sets of rows with coinciding supports are highlighted in red and blue, respectively.

Theorem 7.6. Let $K=\langle a\rangle, L=\langle b\rangle$, and $N=a b N^{\prime}$ with $\operatorname{gcd}(a, b)=1$. Furthermore, let $\varphi \in \mathbb{C}^{N}$ be either the Chu or P4 sequence. Then, the Gabor system $(\varphi, K \times L)$ has the following properties:

(i) $\operatorname{rank}(G)=N$.

(ii) The nonzero eigenvalues of $G$ are $N N^{\prime}$.

In particular, (i) and (ii) together imply that the Gabor system $(\varphi, K \times L)$ is a tight frame with frame bound $N N^{\prime}$.

Proof. (i) We shall show that the support of the first $N$ columns are disjoint, and then conclude that $\operatorname{rank}(G)=N . K$ and $L$ are subgroups of $(\mathbb{Z} / N \mathbb{Z})$ and that $K \cap L=\langle a b\rangle$. Moreover, $G_{m n} \neq 0$ if and only if $\left(\ell_{n}-\ell_{m}\right) \equiv\left(k_{n}-k_{m}\right)$ $\bmod N$. Since $K$ and $L$ are subgroups, this can only happen if the subtractions lie in the intersections of the two groups. That is, $G_{m n} \neq 0$ if and only if

$$
\left(\ell_{n}-\ell_{m}\right) \equiv\left(k_{n}-k_{m}\right) \equiv j a b \bmod N
$$




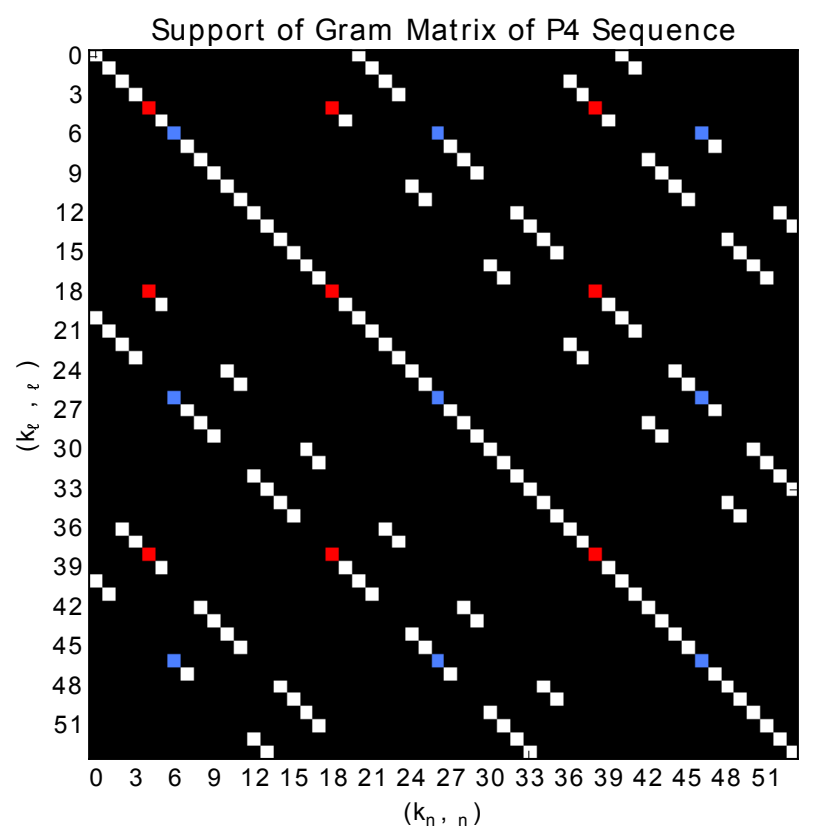

Figure 7.1. Support of $G$ in the case $N=18, \varphi$ is the $\mathrm{P} 4$ sequence, $a=2, b=3$, and $N^{\prime}=3$.

where $j \in\left(\mathbb{Z} / N^{\prime} \mathbb{Z}\right)$. Let $m \in(\mathbb{Z} / N \mathbb{Z})$. By $(7.6)$, we have that for $G_{m n} \neq 0$ we must have that $k_{n}=\left(k_{m}+j a b\right)$ for some $j$. Since $k_{m} \in K$, we have that

$$
k_{n}=\left(j_{m} a+j a b\right)=a\left(j_{m}+j b\right)
$$

for some $j_{m} \in\left(\mathbb{Z} / b N^{\prime} \mathbb{Z}\right)$ and some $j \in\left(\mathbb{Z} / N^{\prime} \mathbb{Z}\right)$.

By the ordering we used for the columns of $G$, we can write the index for column $n$ in terms of $k_{n}$ and $\ell_{n}$ by

$$
n=\left(k_{n} / a\right) a N^{\prime}+\ell_{n} / b=k_{n} N^{\prime}+\ell_{n} / b .
$$

In particular, we would like $n \leq N$, and for that we need that $k_{n}<a b$. Looking at $(7.7)$, we need $\left(j_{m}+j b\right)<b$. There is exactly one such $j \in\left(\mathbb{Z} / N^{\prime} \mathbb{Z}\right)$ which can achieve this and it is obtained by setting $j=-\left\lfloor j_{m} / b\right\rfloor$. Thus, for each row $m$, there is exactly 1 column $n \leq N$ where $G_{m n} \neq 0$, and therefore the first $N$ columns of $G$ are linearly independent. Thus, we conclude $\operatorname{rank}(G) \geq N$. By Lemma 7.1, we know that $\operatorname{rank}(G)=\operatorname{rank}(L)$. Since $L$ is an $M \times N$ matrix, we have that $\operatorname{rank}(G) \leq N$ and we have that $\operatorname{rank}(G)=N$.

(ii) Let $g_{n}$ be the $n$-th column of $G$, with $n \leq N$. We wish to show that $G g_{n}=N N^{\prime} g_{n}$. Note that $G g_{n}[m]$ is given by the inner product of the $m$-th row and the $n$-th column of $G$. Furthermore, since $G$ is self-adjoint, the $n$-th column is also the conjugate of the $n$-th row. If $g_{n}[m] \neq 0$, then $G_{m n} \neq 0$ and $G_{n n} \neq 0$. Thus, by Lemma 7.4 rows $m$ and $n$ have supports that coincide. By Lemma 7.3, 
$G_{m(\cdot)}=C_{m} g_{n}^{*}$, where $\left|C_{m}\right|=1$. Thus, $G g_{n}[m]=C_{m}\left\|g_{n}\right\|_{2}^{2}=N^{2} N^{\prime} C_{m}$. It is easily computed that $G_{n n}=N$, so by Lemma 7.3 , we have that $g_{n}[m]=N C_{m}$. In particular, we get $G g_{n}[m]=\left(N N^{\prime}\right)\left(N C_{m}\right)$. Thus, the first $N$ columns of $G$ are eigenvectors of $G$ and all have eigenvalue $N N^{\prime}$. It now follows that the system is a tight frame with frame bound $N N^{\prime}$.

Remark 7.7. In general, if $\operatorname{gcd}(a, b)>1$, then the above result will not hold. Let $\varphi \in \mathbb{C}^{4}$ be the P4 sequence. That is, $\varphi=(1,-\sqrt{2} / 2-i \sqrt{2} / 2,-1,-\sqrt{2} / 2-$ $i \sqrt{2} / 2)$. Let $K=\{0,2\}$ and $L=\{0,2\}$. Note that this would give $a=b=$ $\operatorname{gcd}(a, b)=2$. The Gabor system $(\varphi, K \times L)$ is given by

$$
\begin{gathered}
(\varphi, K \times L)=\{\pi(0,0) \varphi, \pi(0,2) \varphi, \pi(2,0) \varphi, \pi(2,2) \varphi\} \\
=\left\{\left(\begin{array}{c}
1 \\
-\frac{\sqrt{2}}{2}-\frac{\sqrt{2}}{2} i \\
-1 \\
-\frac{\sqrt{2}}{2}-\frac{\sqrt{2}}{2} i
\end{array}\right),\left(\begin{array}{c}
\frac{\sqrt{2}}{2}+\frac{\sqrt{2}}{2} i \\
-1 \\
\frac{\sqrt{2}}{2}+\frac{\sqrt{2}}{2} i
\end{array}\right),\left(\begin{array}{c}
-1 \\
-\frac{\sqrt{2}}{2}-\frac{\sqrt{2}}{2} i \\
1 \\
-\frac{\sqrt{2}}{2}-\frac{\sqrt{2}}{2} i
\end{array}\right),\left(\begin{array}{c}
-1 \\
\frac{\sqrt{2}}{2}+\frac{\sqrt{2}}{2} i \\
1 \\
\frac{\sqrt{2}}{2}+\frac{\sqrt{2}}{2} i
\end{array}\right),\right\} .
\end{gathered}
$$

Note that the first and fourth vectors are multiples of each other, as well as the second and third vectors. Specifically,

$$
\pi(0,0) \varphi=-\pi(2,2) \varphi \quad \text { and } \quad \pi(0,2) \varphi=-\pi(2,0) \varphi .
$$

We conclude from this that the dimension of the span of the Gabor system $(\varphi, K \times L)$ is only 2 , and the Gabor system in question is not a frame.

We close this section with brief mentions about the proofs in the Wiener sequence case. To simplify further, we only mention the odd length case, but one can easily make the highlighted adjustments in the even case as well.

Lemma 7.8. Let $\varphi \in \mathbb{C}^{N}$ be a Wiener sequence of odd length and let $N=a b N^{\prime}$ where $\operatorname{gcd}(a, b)=1$. Suppose $G$ is the Gram matrix generated by the Gabor system $(\varphi, K \times L)$, where $K=\langle a\rangle$ and $L=\langle b\rangle$. Then, there exist functions $f, g: \mathbb{N} \cup\{0\} \rightarrow \mathbb{C}$ such that $G_{m n} / N=f(n) g(m)$ wherever $G_{m n} \neq 0$.

To prove Lemma 7.8, the same technique used in Lemma 7.3 of writing $k_{n}=$ $c_{n} a b+d_{n} a$ and $k_{m}=c_{m} a b+d_{m} a$ can be used, and the result follows from similar computations to Lemma 7.3.

Lemma 7.9. Let $\varphi \in \mathbb{C}^{N}$ be a Wiener sequence of odd length and let $N=a b N^{\prime}$ where $\operatorname{gcd}(a, b)=1$. Suppose $G$ is the Gram matrix generated by $(\varphi, K \times L)$, where $K=\langle a\rangle$ and $L=\langle b\rangle$. Then, the support of the rows (and columns) of $G$ either completely coincide or are completely disjoint.

To prove Lemma 7.9 , one needs to replace $j a b, j^{\prime} a b, \tilde{j} a b$ in Lemma 7.4 with $2 s j a b, 2 s j^{\prime} a b$, and $2 s \tilde{j} a b$, and the same result will hold.

Theorem 7.10. Let $K=\langle a\rangle, L=\langle b\rangle$, and $N=a b N^{\prime}$ with $\operatorname{gcd}(a, b)=1$. Furthermore, let $\varphi \in \mathbb{C}^{N}$ be a Wiener sequence of odd length. Then, the Gabor system $(\varphi, K \times L)$ has the following properties:

(i) $\operatorname{rank}(G)=N$. 
(ii) The nonzero eigenvalues of $G$ are $N N^{\prime}$.

In particular, (i) and (ii) combined imply that the Gabor system $(\varphi, K \times L)$ is a tight frame with bound $N N^{\prime}$.

As with the modification used in Lemma 7.9, one only needs to change any instance of $j a b$ in Theorem 7.6 with $2 s j a b$ and apply the appropriate computations to prove Theorem 7.10.

\section{Closing remarks}

It is still unclear what the exact role of CAZACs is in the generation of tight frames. Many of the known CAZAC sequences are suitable for generating tight Gabor frames, as shown in Section 5. On the other hand, the Björck sequence is also CAZAC, but is ill-suited for the generation of tight Gabor frames. A large part of the difficulty in further assessing the suitability of CAZAC sequences for generating tight Gabor frames comes from how few CAZAC sequences that we can explicitly write out. Despite several known theorems quantifying how many there are for given length $N$, nearly all known CAZAC sequences are generated by roots of unity. The Björck sequence is, again, the exception to this and is also the one known sequence that is ill-suited for the generation of tight Gabor frames. One could perhaps show that all CAZAC sequences generated by roots of unity (eg. Chu, P4, Wiener, roots of unity generated Milewski) will have sparse discrete periodic ambiguity functions. Hopefully, the discovery of more CAZAC sequences will help clarify the usability of CAZAC sequences in generating tight Gabor frames.

\section{ACKNOWLEDGEMENT}

This work is supported through ARO Grant W911NF-17-1-0014. Special thanks also goes to John Benedetto for this grant opportunity and for being an amazing advisor.

\section{References}

[1] Radu Balan, Peter G. Casazza, Christopher Heil, and Zeph Landau. Density, overcompleteness, and localization of frames. I. Theory. Journal of Fourier Analysis and Applications, 12(2):105-143, 2006.

[2] Radu Balan, Peter G. Casazza, Christopher Heil, and Zeph Landau. Density, overcompleteness, and localization of frames. II. Gabor systems. Journal of Fourier Analysis and Applications, 12(3):307-344, 2006.

[3] John J. Benedetto. Frame decompositions, sampling, and uncertainty principle inequalities. In Wavelets: Mathematics and Applications, chapter 7, pages 247-304. CRC Press, 1993.

[4] John J. Benedetto, Robert L. Benedetto, and Joseph T. Woodworth. Optimal ambiguity functions and Weil's exponential sum bound. Journal of Fourier Analysis and Applications, 18(3):471-487, 2012.

[5] John J. Benedetto, Katherine Cordwell, and Mark Magsino. CAZAC sequences and Haagerup's characterization of cyclic n-roots. pre-print. 
[6] John J. Benedetto and Jeffrey J. Donatelli. Ambiguity function and frame-theoretic properties of periodic zero-autocorrelation waveforms. IEEE Journal of Selected Topics in Signal Processing, 1(1):6-20, 2007.

[7] John J. Benedetto and Matthew Fickus. Finite normalized tight frames. Advances in Computational Mathematics, 18(2-4):357-385, 2003.

[8] John J. Benedetto, Christopher Heil, and David F. Walnut. Gabor systems and the BalianLow theorem. In Gabor Analysis and Algorithms, pages 85-122. Springer, 1998.

[9] John J. Benedetto, Ioannis Konstantinidis, and Muralidhar Rangaswamy. Phase-coded waveforms and their design. IEEE Signal Processing Magazine, 26(1):22-33, 2009.

[10] Göran Björck. Functions of modulus 1 on $\mathbb{Z}_{n}$ whose Fourier transforms have constant modulus and "cyclic $n$-roots". In Recent Advances in Fourier Analysis and its Applications, pages 131-140. Springer, 1990.

[11] Göran Björck and Bahman Saffari. New classes of finite unimodular sequences with unimodular Fourier transforms. Circulant Hadamard matrices with complex entries. Comptes Rendus de l'Académie des Sciences. Série 1, Mathématique, 320(3):319-324, 1995.

[12] Wojciech Bruzda, Wojciech Tadej, and Karol Życzkowski. Complex Hadamard matrices-a catalogue. http://chaos.if.uj.edu.pl/ karol/hadamard/?q=catalogue.

[13] Peter G. Casazza and Jelena Kovačević. Equal-norm tight frames with erasures. Advances in Computational Mathematics, 18(2-4):387-430, 2003.

[14] Peter G. Casazza and Gitta Kutyniok, editors. Finite Frames: Theory and Applications. Birkhäuser Basel, 2013.

[15] Ole Christensen. An Introduction to Frames and Riesz Bases. Applied and Numerical Harmonic Analysis. Springer International Publishing, 2016.

[16] Ingrid Daubechies. The wavelet transform, time-frequency localization and signal analysis. IEEE Transactions on Information Theory, 36(5):961-1005, 1990.

[17] Ingrid Daubechies. Ten Lecutres on Wavelets, volume 61. SIAM, 1992.

[18] Ingrid Daubechies, Bin Han, Amos Ron, and Zuowei Shen. Framelets: MRA-based constructions of wavelet frames. Applied and Computational Harmonic Analysis, 14(1):1-46, 2003.

[19] Richard J. Duffin and Albert C. Schaeffer. A class of nonharmonic Fourier series. Transactions of the American Mathematics Society, 72(2):341-366, 1952.

[20] Gerald Folland and Alladi Sitaram. The uncertainty principle: a mathematical survey. Journal of Fourier Analysis and Applications, 3(3):207-238, 1997.

[21] Dennis Gabor. Theory of communication. part 1: Analysis of information. Electrical Engineers-Part III: Radio and Communiation Engineering, Journal of the Institution of, 93(26):429-441, 1946.

[22] Karlheinz Gröchenig. Foundations of Time-Frequency Analysis. Springer Science \& Business Media, 2013.

[23] Uffe Haagerup. Cyclic p-roots of prime lengths and complex Hadamard matrices. arXiv preprint arXiv:0803.2629, 2008.

[24] A.J.E.M. Janssen. Gabor representation of generalized functions. Journal of Mathematical Analysis and Applications, 83(2):377-394, 1981.

[25] Andrew Kebo, Ioannis Konstantinidis, John J. Benedetto, Michael R. Dellomo, and Jeffrey M. Sieracki. Ambiguity and sidelobe behavior of CAZAC coded waveforms. In 2007 IEEE Radar Conference, pages 99-103. IEEE, 2007.

[26] Jelena Kovačević and Amina Chebira. Life beyond bases: The advent of frames. IEEE Signal Processing Magazine, 2007.

[27] Jelena Kovačević and Amina Chebira. Life beyond bases: The advent of frames (part ii). IEEE Signal Processing Magazine, 5(24):115-125, 2007.

[28] Gitta Kutyniok, Kasso A. Okoudjou, Friedrich Philipp, and Elizabeth K. Tuley. Scalable frames. Linear Algebra and its Applications, 438(5):2225-2238, 2013. 
[29] Jim Lawrence, Götz Pfander, and David Walnut. Linear independence of Gabor systems in finite dimensional vector spaces. Journal of Fourier Analysis and Applications, 11(6):715$726,2005$.

[30] Nadav Levanon and Eli Mozeson. Radar Signals. John Wiley \& Sons, 2004.

[31] Yurii Lyubarskii. Frames in the Bargmann space of entire functions. Entire and Subharmonic Functions, 11:167-180, 1992.

[32] Stéphane Mallat. A Wavelet Tour of Signal Processing. Academic press, 1999.

[33] Andrzej Milewski. Periodic sequences with optimal properties for channel estimation and fast start-up equalization. IBM Journal of Research and Development, 27(5):426-431, 1983.

[34] Dustin Mixon. Sparse signal processing with frame theory. arXiv preprint arXiv:1204.5958, 2012.

[35] Götz Pfander. Gabor frames in finite dimensions. In Finite Frames, pages 193-239. Springer, 2013.

[36] Götz Pfander and Holger Rauhut. Sparsity in time-frequency representations. Journal of Fourier Analysis and Applications, 16(2):233-260, 2010.

[37] Götz Pfander, Holger Rauhut, and Joel A. Tropp. The restricted isometry property for time-frequency structured random matrices. Probability Theory and Related Fields, 156(34):707-737, 2013.

[38] Götz Pfander and David Walnut. Measurement of time-variant linear channels. IEEE Transactions on Information Theory, 52(11):4808-4820, 2006.

[39] Thomas Strohmer. Approximation of dual Gabor frames, window decay, and wireless communications. Applied and Computational Harmonic Analysis, 11(2):243-262, 2001.

[40] Richard Vale and Shayne Waldron. Tight frames and their symmetries. Constructive Approximation, 21(1):83-112, 2004.

[41] Richard Vale and Shayne Waldron. Tight frames generated by finite nonabelian groups. Numerical Algorithms, 48(1-3):11-27, 2008.

[42] David F. Walnut. Weyl-Heisenberg Wavelet Expansions: Existance and Stability in Weighted Spaces. PhD thesis, University of Maryland, 1989. 